\title{
High-Cost Patients and Preventable Spending: A Population-Based Study
}

\author{
Claire de Oliveira, PhDa,b,c; Joyce Cheng, MSc'; Kelvin Chan, MD, PhD, FRCPCd; Craig C. Earle, MD, MSc, FRCPCe; \\ Murray Krahn, MD, MSc, FRCPC; ; and Nicole Mittmann, PhDg
}

\section{ABSTRACT}

Background: Although high-cost $(\mathrm{HC})$ patients make up a small proportion of patients, they account for most health system costs. However, little is known about HC patients with cancer or whether some of their care could potentially be prevented. This analysis sought to characterize $\mathrm{HC}$ patients with cancer and quantify the costs of preventable acute care (emergency department visits and inpatient hospitalizations). Methods: This analysis examined a populationbased sample of all HC patients in Ontario in 2013. HC patients were defined as those above the 90th percentile of the cost distribution; all other patients were defined as non-high-cost (NHC). Patients with cancer were identified through the Ontario Cancer Registry. Sociodemographic and clinical characteristics were examined and the costs of preventable acute care for both groups by category of visit/condition were estimated using validated algorithms. Results: Compared with NHC patients with cancer $(n=369,422), \mathrm{HC}$ patients with cancer $(n=187,770)$ were older (mean age 70 vs 65 years), more likely to live in low-income neighborhoods (19\% vs $16 \%)$, sicker, and more likely to live in long-term care homes ( $8 \%$ vs $0 \%$ ). Although most patients from both cohorts tended to be diagnosed with breast, prostate, or colorectal cancer, those with multiple myeloma or pancreatic or liver cancers were overrepresented among the $\mathrm{HC}$ group. Moreover, $\mathrm{HC}$ patients were more likely to have advanced cancer at diagnosis and be in the initial or terminal phase of treatment compared with $\mathrm{NHC}$ patients. Among $\mathrm{HC}$ patients with cancer, $9 \%$ of spending stemmed from potentially preventable/avoidable acute care, whereas for NHC patients, this spending was approximately $30 \%$. Conclusions: HC patients with cancer are a unique subpopulation. Given the type of care they receive, there seems to be limited scope to prevent acute care spending among this patient group. To reduce costs, other strategies, such as making hospital care more efficient and generating less costly encounters involving chemotherapy, should be explored.

J Natl Compr Canc Netw 2020;18(1):23-31 doi: $10.6004 /$ jnccn.2019.7342

anstitute for Mental Health Policy Research, Centre for Addiction and Mental Health, Toronto; 'bnstitute of Health Policy, Management and Evaluation, University of Toronto, Toronto; 'Institute for Clinical Evaluative Sciences, Toronto; ${ }^{d}$ Odette Cancer Centre, Sunnybrook Health Sciences Centre, Toronto;

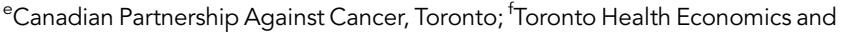
Technology Assessment Collaborative, Toronto; and ${ }^{9}$ Cancer Care Ontario, Toronto, Ontario, Canada.

\section{Background}

Recent publications have shown that patients with cancer incur high costs of care, particularly after diagnosis and in the last year of life. ${ }^{1-3}$ Given the increasing number of patients with cancer ${ }^{4}$ and increasing treatment costs, ${ }^{5}$ policymakers are continually seeking ways to bend the cost curve without sacrificing the quality of care. Focusing on high-cost (HC) patients is likely to have a large impact on health system costs, because these patients account for most healthcare spending. Many jurisdictions have implemented high-risk care management strategies to reduce costs and improve quality. However, it is not clear which costs can be reduced, especially among patients who require costly care, such as those with cancer. One potential solution to decrease costs without sacrificing care is to target preventable or avoidable acute care-that is, acute care for which good outpatient care could likely prevent the need for emergency department (ED) visits and/or hospitalization. Previous research has shown that ED visits and hospitalizations make up more than half of all costs among HC patients. ${ }^{6}$ Furthermore, some studies have shown that a substantial proportion of ED visits ${ }^{7,8}$ and hospitalizations $\mathrm{s}^{9-12}$ may be prevented.

Little is known about HC patients with cancer and their healthcare utilization and spending patterns. Previous work has shown that individuals with cancer make up $20 \%$ of all HC patients. ${ }^{13}$ This is not surprising given that the population is aging, the number of patients diagnosed with cancer is increasing, ${ }^{4}$ and the introduction of newer and more expensive treatments has contributed to an increase in treatment costs. ${ }^{5}$ Wodchis et $\mathrm{al}^{14}$ examined the cost trajectories of patients with cancer over time and identified patient and system characteristics associated with high system costs after cancer treatment. The most common trajectory consisted of patients who were low-cost in the year before cancer treatment and remained low-cost after completing cancer treatment. In addition, the investigators

See JNCCN.org for supplemental online content. 
found that increases in age and multimorbidity and low continuity of care were the strongest predictors of high costs after cancer treatment. Lam et $\mathrm{al}^{15}$ examined Medicare data for $\mathrm{HC}$ and non-high-cost (NHC) patients with and without cancer in 2014, and found that despite $15 \%$ of Medicare patients having a cancer diagnosis, the prevalence of cancer was higher among HC patients $33 \%$ vs $13 \%)$. Furthermore, the investigators found that HC patients had 3 times greater odds of having cancer and higher total annual spending ( $\$ 66,685$ vs $\$ 59,427$ ).

However, it is not clear which costs, if any, can be reduced for this patient population. Joynt et $\mathrm{al}^{16}$ examined preventable and nonpreventable acute care spending among HC Medicare patients using validated algorithms and found that only a small percentage of costs resulted from preventable acute care. Graven et al ${ }^{17}$ replicated that analysis, using Oregon's All Payer All Claims database and Medicaid data from the Oregon Health Authority, and found that preventable acute care spending for HC patients accounted for $<6 \%$ of their total spending. Using data from Canada, Ronksley et al ${ }^{18}$ examined preventable acute care spending among hospitalized HC patients (at the Ottawa Hospital) using an ambulatory care-sensitive conditions algorithm. They found that among the HC inpatient population, most costs were due to a single nonpreventable acute care episode. Despite this work, none of these studies specifically examined HC subgroups, such as patients with cancer.

The objectives of this analysis were to provide an in-depth characterization of HC patients diagnosed with cancer, while comparing them to NHC patients with cancer, and to quantify the costs of potentially preventable/avoidable acute care (ie, ED visits and inpatient hospitalizations).

\section{Methods}

\section{Data}

We used administrative healthcare data available through ICES (Institute for Clinical Evaluative Sciences) in Toronto, Ontario, which includes individual-level linkable data on most publicly funded healthcare services for all legal residents of Ontario, Canada's most populous province. ${ }^{19}$ Data on inpatient care are captured in the Discharge Abstract Database, the Ontario Mental Health Reporting System, the Continuing Care Reporting System, and the National Rehabilitation Reporting System, whereas data on ambulatory care are recorded in the National Ambulatory Care Reporting System. The Ontario Health Insurance Plan claims database captures data on physician visits, including fee-forservice visits and shadow-billed services, and laboratory claims. The Ontario Drug Benefit program database includes information on all outpatient prescription drugs dispensed to individuals eligible for public drug coverage (ie, individuals on social assistance and those aged $\geq 65$ years). The Home Care Database records all unique visits by home care providers. The Ontario Cancer Registry provides data on all patients diagnosed with a malignant neoplasm in Ontario. The ICES data repository also includes other databases, which provide useful sociodemographic data on patients. The Registered Persons Database, a population-based registry, provides basic demographic data (such as age and sex) on all legal residents of Ontario. The Immigration, Refugees, and Citizenship Canada database provides information on all legal immigrants and refugees in Canada. Census data, obtained through Statistics Canada, contain information on neighborhood-level data, such as income and rurality. All databases were linked using unique encoded identifiers and analyzed at ICES, in compliance with Ontario's privacy legislation. A full description of the databases can be found in supplemental eTable 1, available with this article at JNCCN.org.

\section{Patient Cohorts}

We selected all adult patients (aged $\geq 18$ years) eligible for public healthcare insurance and residing in Ontario in 2013. All individuals who did not contact the Ontario healthcare system in 2013 and those who died during 2013 (but not those in their last year of life) were excluded, in line with previous research, ${ }^{16}$ because assessing the preventability of end-of-life care costs was beyond the scope of this analysis.

HC patients were defined as those above the 90th percentile of the cost distribution (ie, the top decile) in 2013 , in line with previous work. ${ }^{20}$ All other patients were defined as NHC patients. This threshold provided a larger cohort of patients compared with other thresholds. Within each group, we identified those ever diagnosed with cancer (before or during 2013) through use of the Ontario Cancer Registry. To estimate healthcare costs and thus determine patients with costs above the 90th percentile, we used a cost estimation algorithm, available at ICES. ${ }^{19}$ Healthcare costs included all costs borne by the public third-party payer (ie, the Ontario Ministry of Health and Long-Term Care) ${ }^{19}$ : inpatient hospitalizations (both psychiatric and acute), other institution-based care (inpatient rehabilitation, complex continuing care, long-term care), ED visits and other ambulatory care, outpatient clinic visits, physician visits and outpatient care, outpatient prescription drugs (for individuals covered under the public provincial drug plan), and home care. Costs captured by the algorithm accounted for $>90 \%$ of all government-paid healthcare costs. ${ }^{21}$ 
Identifying Preventable Acute Care

To identify preventable ED visits, we used an updated version of a validated algorithm created at NYU Wagner ${ }^{22}$ by John Billings and colleagues and used in prior research examining HC Medicare patients. ${ }^{16}$ The NYU algorithm uses specified diagnosis codes to determine the following type of ED visits: nonemergent, emergent but primary care treatable, emergent ED care needed but preventable, and emergent ED care needed and not preventable. The updated version of the algorithm includes additional categories for injury, mental health, alcohol use, and drug use. $^{23}$ Based on these classifications, Billings et $\mathrm{al}^{24}$ compiled a set of probabilistic weights that were applied to ED discharge data using the primary discharge ICD-10 diagnosis codes to determine the percentage of ED use attributable to each category. ED visits with diagnosis codes not mapped to any of the 8 categories were assigned to the "unclassified" category. Preventable ED visits were defined as nonemergent, emergent/primary care treatable, and emergent/ED care needed but preventable, in line with prior work. ${ }^{16}$

To identify potentially preventable hospitalizations, we used a validated algorithm, Quality Prevention Quality Indicators, developed by the Agency for Healthcare Research and Quality ${ }^{25}$ and used elsewhere. ${ }^{26-28}$ This algorithm defines potentially preventable hospitalizations as those related to conditions, such as heart failure, diabetes, hypertension, and asthma, for which good outpatient care can likely prevent the need for hospitalization. In addition, we used the respective prevention quality indicators for common nonpreventable clinical diagnosis groups to identify nonpreventable hospitalizations (see supplemental eAppendix 1 for codes).

\section{Analysis}

\section{Patient Descriptives}

HC and NHC patients with cancer were characterized and compared in terms of sociodemographic (sex, age, migrant status, neighborhood-level income quintile, urban/rural residence) and clinical characteristics (chronic conditions, frailty, residence in a long-term care home). We used the existing ICES-derived cohorts/registries to ascertain chronic conditions among patients ${ }^{29-40}$ and the Johns Hopkins Adjusted Clinical Groups Case-Mix System $^{41}$ to obtain data on patient frailty. Residence in a longterm care facility was determined through the Continuing Care Reporting System. Using information included in the Ontario Cancer Registry, we also characterized patient groups by cancer type (solid vs hematologic) and site, cancer stage at diagnosis (where available), treatment phase (initial, continuing, terminal) ${ }^{1,2}$ and days since first diagnosis (patients with multiple cancers were assigned

Table 1. Sociodemographic Characteristics of Patient Cohorts

\begin{tabular}{|c|c|c|c|c|}
\hline & $\begin{array}{l}\text { High-Cost Patients } \\
\text { n (\%) }\end{array}$ & $\begin{array}{c}\text { Non-High-Cost Patients } \\
\text { n (\%) }\end{array}$ & $P$ Value & $\begin{array}{l}\text { All Patients } \\
\text { n (\%) }\end{array}$ \\
\hline Total, N & 187,770 & 369,422 & & 557,192 \\
\hline Female & $98,001(52.2)$ & $202,213(54.7)$ & & $300,214(53.9)$ \\
\hline Male & $89,769(47.8)$ & $167,209(45.3)$ & & $256,978(46.1)$ \\
\hline Nonimmigrant & $173,622(92.5)$ & $337,369(91.3)$ & $<.001$ & $510,991(91.7)$ \\
\hline Immigrant & $11,969(6.4)$ & $27,492(7.4)$ & $<.001$ & $39,461(7.1)$ \\
\hline Refugee & $2,179(1.2)$ & $4,561(1.2)$ & .017 & $6,740(1.2)$ \\
\hline \multicolumn{5}{|c|}{ Neighborhood income quintile } \\
\hline 3rd, medium & $36,834(19.6)$ & $72,885(19.7)$ & .316 & $109,719(19.7)$ \\
\hline 4th, medium-high & $38,539(20.5)$ & $80,614(21.8)$ & $<.001$ & $119,153(21.4)$ \\
\hline 5th, high & $38,678(20.6)$ & $86,983(23.5)$ & $<.001$ & $125,661(22.6)$ \\
\hline Rural residence & & & .281 & \\
\hline No & $162,412(86.5)$ & $319,146(86.4)$ & & $481,558(86.4)$ \\
\hline Yes & $25,358(13.5)$ & $50,276(13.6)$ & & $75,634(13.6)$ \\
\hline
\end{tabular}


to the first cancer diagnosed; see supplemental eTable 2 for ICD-O codes). Patient characteristics between HC and NHC individuals with cancer were compared using a chi-square test for differences.

\section{Costs of Preventable Acute Care Among HC Patients}

We estimated the total costs of preventable and nonpreventable acute care by category of visit (for ED visits) and condition (for inpatient hospitalizations) for $\mathrm{HC}$ and NHC patients with cancer. All costs were reported in 2016 constant Canadian dollars using the Statistics Canada Consumer Price Index for healthcare. ${ }^{42}$

All analyses were performed using SAS Enterprise Guide 7.1 (SAS Institute Inc). The study was approved by the research ethics board of Sunnybrook Health Sciences Centre.

\section{Results}

\section{Patient Characteristics}

In 2013, 10,031,865 individuals contacted the Ontario healthcare system; 1,003,187 were defined as HC patients, and the remainder were defined as NHC $(n=9,028,678)$. After excluding all patients who died during 2013 $(\mathrm{n}=90,555)$, we were left with 929,726 HC and 9,011,584 NHC patients. Across both groups, 557,192 patients had cancer; 187,770 were HC and 369,422 were NHC.

HC patients with cancer were older (mean age, 69.9 vs 64.5 years) and slightly more represented in the lower neighborhood income quintile (19.0\% vs $15.6 \%)$ compared with NHC patients, but the 2 groups were roughly the same for all other sociodemographic characteristics (Table 1). HC patients with cancer had a higher proportion of all chronic conditions examined, particularly HIV, chronic heart failure, and dementia. HC patients with cancer also had a higher degree of frailty and were more likely to be living in long-term care facilities (Table 2). Among all patients, most were diagnosed with breast, prostate, and colorectal cancers (Table 3). However, multiple myeloma and pancreatic and liver cancers were overrepresented among HC patients, whereas testicular, thyroid, and cervical cancers and melanoma were overrepresented among NHC patients. Moreover, although most patients had missing data on cancer stage, a higher proportion of patients with stages III and IV was seen in the HC group. There was also a higher proportion of patients in the initial and terminal phases of treatment among the HC group (note that the terminal phase did not include patients who died but rather those in the last year of life, for whom death likely occurred in 2014). HC patients with cancer also had a shorter length of time since first diagnosis $(2,917.9$ vs $3,844.6$ days).

\section{Costs of Preventable and Nonpreventable Acute Care}

\section{ED Visits}

Within the HC cohort, $30.8 \%$ of the ED costs were classified as preventable (Table 4). Patterns were somewhat

Table 2. Clinical Characteristics of Patient Cohorts

\begin{tabular}{|c|c|c|c|c|}
\hline & $\begin{array}{l}\text { High-Cost Patients } \\
\text { n (\%) }\end{array}$ & $\begin{array}{c}\text { Non-High-Cost Patients } \\
\text { n (\%) }\end{array}$ & $P$ Value & $\begin{array}{l}\text { All Patients } \\
\text { n (\%) }\end{array}$ \\
\hline Total, N & 187,770 & 369,422 & & 557,192 \\
\hline \multicolumn{5}{|l|}{ Chronic conditions } \\
\hline Arthritis & $5,862(3.1)$ & $5,807(1.6)$ & $<.001$ & $11,669(2.1)$ \\
\hline Asthma & $30,945(16.5)$ & $45,438(12.3)$ & $<.001$ & $76,383(13.7)$ \\
\hline COPD & $53,878(28.7)$ & $58,047(15.7)$ & $<.001$ & $111,925(20.1)$ \\
\hline Diabetes & $62,963(33.5)$ & $78,738(21.3)$ & $<.001$ & $141,701(25.4)$ \\
\hline HIV & $869(0.5)$ & $396(0.1)$ & $<.001$ & $1,265(0.2)$ \\
\hline Hypertension & $131,178(69.9)$ & $201,941(54.7)$ & $<.001$ & $333,119(59.8)$ \\
\hline Chronic heart failure & $30,310(16.1)$ & $16,657(4.5)$ & $<.001$ & $46,967(8.4)$ \\
\hline Crohn's disease/colitis & $2,505(1.3)$ & $3,227(0.9)$ & $<.001$ & $5,732(1.0)$ \\
\hline Chronic kidney disease & $586(0.3)$ & $163(0.0)$ & $<.001$ & $749(0.1)$ \\
\hline Myocardial infarction & $13,121(7.0)$ & $11,209(3.0)$ & $<.001$ & $24,330(4.4)$ \\
\hline Psychosis & $5,443(2.9)$ & $3,380(0.9)$ & $<.001$ & $8,823(1.6)$ \\
\hline Dementia & $23,223(12.4)$ & $8,566(2.3)$ & $<.001$ & $31,789(5.7)$ \\
\hline \multicolumn{5}{|l|}{ Comorbidity } \\
\hline Frailty & $28,157(15.0)$ & $6,827(1.8)$ & $<.001$ & $34,984(6.3)$ \\
\hline Long-term care & $14,328(7.6)$ & $45(0.0)$ & $<.001$ & $14,373(2.6)$ \\
\hline
\end{tabular}

Abbreviation: COPD, chronic obstructive pulmonary disease. 


\section{Table 3. Cancer-Specific Characteristics of Study Cohorts}

\begin{tabular}{|c|c|c|c|c|}
\hline & $\begin{array}{l}\text { High-Cost Patients } \\
\text { n (\%) }\end{array}$ & $\begin{array}{c}\text { Non-High-Cost Patients } \\
n(\%)\end{array}$ & $P$ Value & $\begin{array}{l}\text { All Patients } \\
\text { n (\%) }\end{array}$ \\
\hline Total, N & 187,770 & 369,422 & & 557,192 \\
\hline Hematologic & $18,703(10.0)$ & $30,319(8.3)$ & & 49,022 (8.8) \\
\hline Solid & $169,067(90.0)$ & $339,103(91.7)$ & & $508,170(91.2)$ \\
\hline Esophageal & $810(0.4)$ & $620(0.2)$ & $<.001$ & $1,430(0.3)$ \\
\hline Pancreatic & $1,299(0.7)$ & $809(0.2)$ & $<.001$ & $2,108(0.4)$ \\
\hline Colorectal & $23,506(12.5)$ & $36,867(10.0)$ & $<.001$ & $60,373(10.8)$ \\
\hline Liver & $1,188(0.6)$ & $747(0.2)$ & $<.001$ & $1,935(0.3)$ \\
\hline Melanoma & $7,666(4.1)$ & $27,334(7.4)$ & $<.001$ & $35,000(6.3)$ \\
\hline Female breast & $36,402(19.4)$ & $77,540(21.0)$ & $<.001$ & $113,942(20.4)$ \\
\hline Cervical & $2,062(1.1)$ & $7,698(2.1)$ & $<.001$ & $9,760(1.8)$ \\
\hline Uterine corpus & $7,617(4.1)$ & $16,827(4.6)$ & $<.001$ & $24,444(4.4)$ \\
\hline Ovarian & $2,840(1.5)$ & $5,294(1.4)$ & .019 & $8,134(1.5)$ \\
\hline Prostate & $32,688(17.4)$ & $68,391(18.5)$ & $<.001$ & $101,079(18.1)$ \\
\hline Testicular & $1,021(0.5)$ & $6,850(1.9)$ & $<.001$ & $7,871(1.4)$ \\
\hline Kidney & $5,634(3.0)$ & $10,170(2.8)$ & $<.001$ & $15,804(2.8)$ \\
\hline Bladder & $7,499(4.0)$ & $9,059(2.5)$ & $<.001$ & $16,558(3.0)$ \\
\hline Missing & $108,951(58.0)$ & $258,592(70.0)$ & $<.001$ & $367,543(66.0)$ \\
\hline 0 & $301(0.2)$ & $651(0.2)$ & .174 & $952(0.2)$ \\
\hline I & $25,332(13.5)$ & $46,617(12.6)$ & $<.001$ & $71,949(12.9)$ \\
\hline II & $26,859(14.3)$ & $42,731(11.6)$ & $<.001$ & $69,590(12.5)$ \\
\hline III & $16,132(8.6)$ & $15,773(4.3)$ & $<.001$ & $31,905(5.7)$ \\
\hline IV & $9,485(5.1)$ & $4,326(1.2)$ & $<.001$ & $13,811(2.5)$ \\
\hline Unknown/Occult & $710(0.4)$ & $732(0.2)$ & $<.001$ & $1,442(0.3)$ \\
\hline \multicolumn{5}{|l|}{ Treatment phase } \\
\hline Initial ${ }^{\mathrm{b}}$ & $46,822(24.9)$ & $16,829(4.6)$ & $<.001$ & $63,651(11.4)$ \\
\hline Continuing & $120,568(64.2)$ & $347,674(94.1)$ & $<.001$ & $468,242(84.0)$ \\
\hline Terminal $^{c}$ & $20,380(10.9)$ & $4,919(1.3)$ & $<.001$ & $25,299(4.5)$ \\
\hline Days since first diagnosis, mean (SD) & $2,917.9(3,188.8)$ & $3,844.6(3,008.7)$ & $<.001$ & $3,532.3(3,101.7)$ \\
\hline
\end{tabular}

aSee supplemental eTable 2 for the ICD-O and histology codes used to define each cancer site.

bDefined as the 6-month period after date of diagnosis.

'Defined as the 12-month period before date of death. 


\begin{tabular}{|c|c|c|c|}
\hline & $\begin{array}{l}\text { High-Cost Patients } \\
\text { CAD\$ }(\%)\end{array}$ & $\begin{array}{c}\text { Non-High-Cost Patients } \\
\text { CAD\$ (\%) }\end{array}$ & $\begin{array}{l}\text { All Patients } \\
\text { CAD\$ (\%) }\end{array}$ \\
\hline \multicolumn{4}{|l|}{ Category of visit } \\
\hline Not emergent $(A)$ & $12,823,306(10.7)$ & $4,445,660(14.9)$ & $17,268,966(11.6)$ \\
\hline Emergent, primary care treatable (B) & $15,811,564(13.2)$ & $4,625,172(15.5)$ & $20,436,736(13.7)$ \\
\hline ED care need, preventable (C) & $8,222,187(6.9)$ & $1,456,994(4.9)$ & $9,679,181(6.5)$ \\
\hline ED care needed, nonpreventable & $23,931,199(20.1)$ & $4,090,213(13.8)$ & $28,021,412(18.8)$ \\
\hline Alcohol-related & $337,171(0.3)$ & $127,048(0.4)$ & $464,219(0.3)$ \\
\hline Drug-related & $71,716(0.1)$ & $21,964(0.1)$ & $93,680(0.1)$ \\
\hline Injury & $13,673,609(11.5)$ & $4,873,770(16.4)$ & $18,547,379(12.4)$ \\
\hline Mental health-related & $1,936,973(1.6)$ & $417,858(1.4)$ & $2,354,831(1.6)$ \\
\hline Other/Unclassified & $42,546,957(35.6)$ & $9,694,866(32.6)$ & $52,241,823(35.0)$ \\
\hline Total preventable $(\mathrm{A}+\mathrm{B}+\mathrm{C})$ & $36,857,057(30.9)$ & $10,527,826(35.4)$ & $47,384,883(31.8)$ \\
\hline Total nonpreventable & $39,950,668(33.5)$ & $9,530,853(32.0)$ & $49,481,521(33.2)$ \\
\hline Not classified & $42,546,957(35.6)$ & $9,694,866(32.6)$ & $52,241,823(35.0)$ \\
\hline Total & $119,354,682(100)$ & $29,753,545(100)$ & $149,108,227(100)$ \\
\hline
\end{tabular}

Abbreviation: ED, emergency department.

similar for the NHC cohort, with $35.3 \%$ of costs deemed preventable. Emergent, primary care treatable ED visits made up the highest proportion of preventable care costs for both HC and NHC patients $13.2 \%$ and $15.5 \%$, respectively). However, HC patients had a higher proportion of nonpreventable ED visit costs than NHC patients ( $20.1 \%$ vs $13.8 \%)$. In addition, costs of ED visits with injuries represented a relatively high proportion among NHC patients (16.4\%).

\section{Inpatient Care}

HC patients with cancer had a higher proportion of preventable inpatient costs than NHC patients $(7.3 \%$ vs $6.6 \%$ ) (Table 5). The most common reasons for preventable hospitalizations among $\mathrm{HC}$ patients with cancer included chronic obstructive pulmonary disease (COPD), bacterial pneumonia, and urinary tract infections; this was also the case for NHC patients (but with different rankings). In contrast, the most common reasons for nonpreventable hospitalizations were cancer and chemotherapy (28.1\%), orthopedic conditions $(5.0 \%)$, and ischemic heart disease (4.0\%) for HC patients, and cancer and chemotherapy (13.8\%), gastrointestinal infections and disorders (10.7\%), and syncope and dizziness (3.3\%) among NHC patients (data not shown).

Combining both acute settings, $9.0 \%$ of HC patients' costs were considered potentially preventable compared with $29.9 \%$ for HNC patients.

\section{Discussion}

We examined a population-based sample of HC patients with cancer and estimated potentially preventable acute care spending while comparing them with NHC patients with cancer. HC patients were older, slightly more represented in the lowest neighborhood income quintile, sicker, and more likely to live in long-term care homes compared with NHC patients. HC patients were also more likely to be diagnosed with high-mortality cancers, such as multiple myeloma and pancreatic and liver cancers, and to have an advanced stage at diagnosis. Moreover, HC patients were more likely to be in the initial and terminal phases of treatment and to have a shorter length of time since first diagnosis. Roughly $30.9 \%$ of ED visit costs and $7.3 \%$ of hospitalization costs among HC patients could potentially be avoided, in contrast to roughly $35.4 \%$ and $6.6 \%$, respectively, for NHC patients. The top categories for preventable care were primary care-treatable ED visits and hospitalizations for COPD, pneumonia, and urinary tract infections. Combining both acute settings, $9.0 \%$ of HC patients' costs were considered potentially preventable, whereas the corresponding value for NHC patients was more than 3 times higher (29.9\%).

Previous research has found that patients with high-mortality cancers have higher treatment costs. ${ }^{43}$ This is also the case for patients with advanced cancer ${ }^{1}$ and those in the initial and terminal phases of treatment. ${ }^{1,2,44,45}$ Moreover, another study on HC patients showed that long-term care residence has a large impact on costs. ${ }^{46}$ Therefore, it is not surprising that patients with these characteristics were more often in the HC category.

Some research has examined the relationship between being an HC patient and having cancer. For example, 


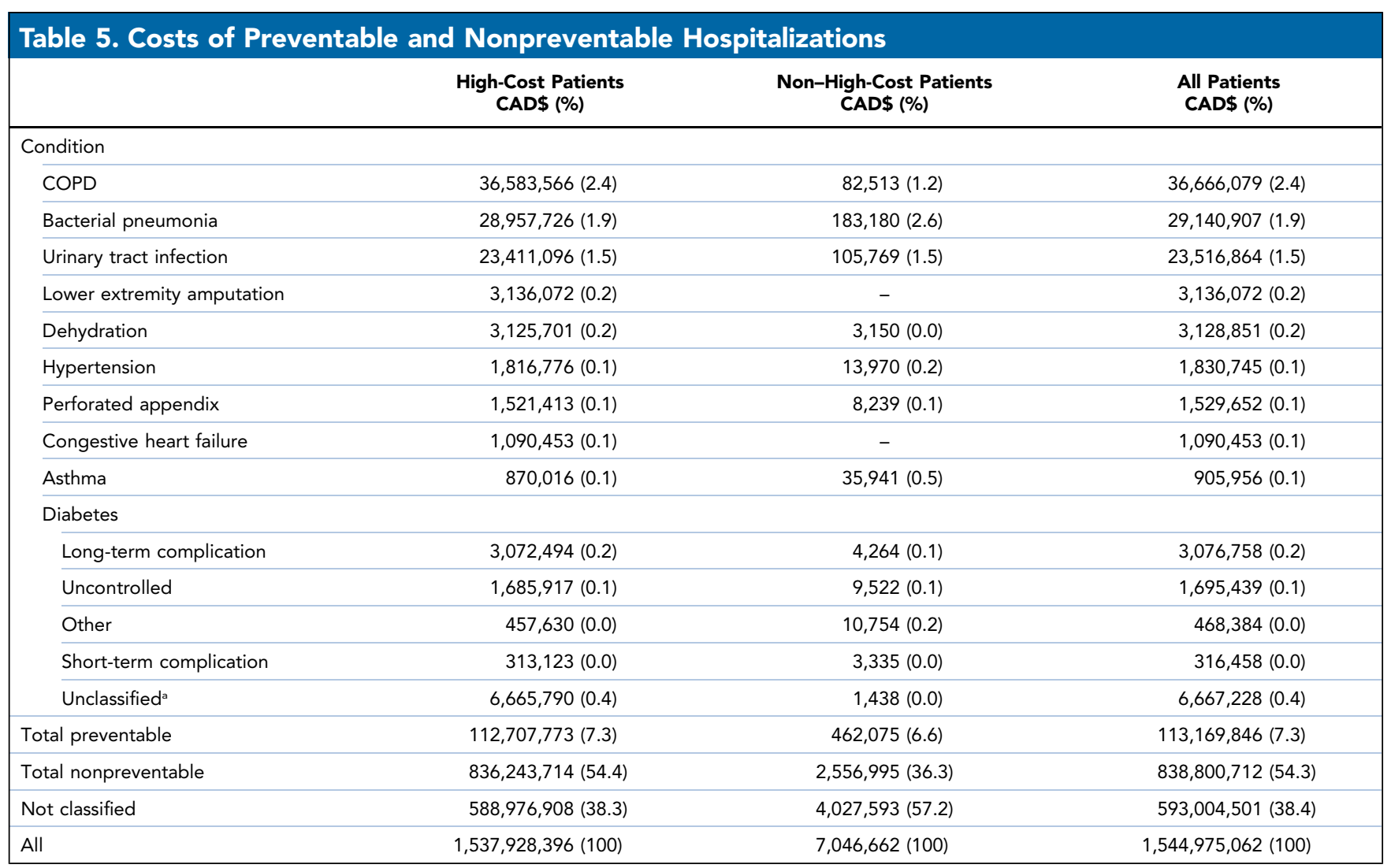

Abbreviation: COPD, chronic obstructive pulmonary disease.

ancludes all diabetes codes that do not fall in any of the other existing diabetes categories.

Wodchis et $\mathrm{al}^{14}$ examined the cost trajectories of patients with cancer over time to understand the predictors of being an HC patient after a cancer diagnosis. The most common trajectory was low-cost in the years before and after cancer treatment (31.4\%). The next most common trajectory, which accounted for nearly $15 \%$ of cases examined, included patients who were low-cost before diagnosis but HC in the year after cancer treatment ended, suggesting that cancer plays a role in becoming a $\mathrm{HC}$ patient. Lam et $\mathrm{al}^{15}$ examined Medicare data for HC and NHC patients with and without cancer in 2014 and found that the prevalence of cancer was higher among HC patients (33\% vs $13 \%$ ) and that these patients had 3 times greater odds of having cancer and higher total annual spending. No existing studies have specifically examined preventable acute care among specific HC subgroups, such as HC patients with cancer; most of the literature on this topic has examined all $\mathrm{HC}$ patients and found that only a small percentage of costs were due to preventable acute care. ${ }^{13,16,17}$ We also found this to be the case for HC patients with cancer. In particular, we found that only $9 \%$ of acute care costs were considered preventable, which is close to what other researchers have found in the United States, albeit for the entire HC population.
These results suggest that overall, among HC patients with cancer, there is limited scope to prevent acute care costs. However, a greater opportunity may exist to prevent these costs among some types of cancer and/or cancer sites. For example, despite small differences, HC patients with hematologic cancers had a slightly higher proportion of preventable acute care costs than those with solid cancers (data not reported). Future work should seek to undertake this analysis by cancer type/site. Despite the fact that more than one-third of acute care costs were for healthcare encounters that could not be classified, a large proportion of costs were for nonpreventable acute care. In particular, the largest proportion of nonpreventable hospitalization costs was for episodes of care involving chemotherapy, which is standard treatment for many patients with cancer. Chemotherapy costs tend to represent a large proportion of treatment costs, and these costs have been increasing over time. ${ }^{5}$ Thus, the ability to lower acute care costs through better outpatient care may be limited. Nonetheless, there may be instances when chemotherapy may be avoided ${ }^{47}$ or deemed unnecessary, ${ }^{48}$ despite these hospitalizations being defined as nonpreventable by the algorithm. Moreover, costs could potentially be reduced 
by making hospital care more efficient so that each inpatient care episode is less costly, ${ }^{16}$ and through less costly encounters involving chemotherapy.

Strengths of our study include its use of a population-based sample of all adult HC patients with cancer in Ontario, which is Canada's most populous province, whereas many existing studies tend to be limited to older populations. ${ }^{15,16}$ Additionally, we accounted for all costs associated with ED visits and hospitalization, given the presence of a (sole) third-party public payer for acute care in Ontario. We provided an in-depth characterization of the HC patient population with cancer and quantified the costs of potentially preventable acute care.

Nonetheless, this study is not without limitations. We did not examine patients aged $<18$ years, because these patients typically have different cancers compared with adults and their cancer-related care is organized differently compared with adults. We also did not examine the preventability of end-of-life care, because this was outside the scope of the analysis. Additionally, data on cancer stage were missing for most patients during the analysis period. Lastly, we used algorithms developed in the United States to identify preventable acute care; however, these algorithms could not classify all visits, and considered all ED visits for mental health and alcohol and drug use as nonpreventable, although it is likely that some of these visits could potentially be preventable. Furthermore, these algorithms are not disease-specific and thus did not examine preventable cancer-specific acute care; future work should explore this area and what constitutes potentially preventable cancer-specific acute care.

\section{Conclusions}

HC patients with cancer are a unique subpopulation within the HC patient population, with specific needs and healthcare utilization patterns. Given the care they receive, there seems to be limited scope to prevent acute care spending. To reduce costs, other strategies should be explored, such as making hospital care more efficient and ensuring encounters involving chemotherapy are less costly.

Submitted January 8, 2019; accepted for publication July 26, 2019.

Author contributions: Study concept and design: de Oliveira, Chan, Earle, Krahn, Mittmann. Data acquisition: Cheng. Data analysis and interpretation: All authors. Project management: de Oliveira. Manuscript preparation: de Oliveira. Critical revision: Cheng, Chan, Earle, Krahn, Mittmann.

Disclosures: The authors have not received any financial consideration from any person or organization to support the preparation, analysis, results, or discussion of this article.

Funding and disclaimers: This study was conducted with the support of Cancer Care Ontario (CCO) through funding provided by the Government of Ontario. Parts of this material are based on data and information provided by CCO. The opinions, results, view, and conclusions reported in this article are those of the authors and do not necessarily reflect those of $\mathrm{CCO}$. No endorsement by $\mathrm{CCO}$ is intended or should be inferred. This study was also supported by the Institute for Clinical Evaluative Sciences (ICES), which is funded by an annual grant from the Ontario Ministry of Health and Long-Term Care (MOHLTC). The opinions, results, and conclusions reported in this article are those of the authors and are independent from the funding sources. No endorsement by ICES or the Ontario MOHLTC is intended or should be inferred. Parts of this material are based on data and/or information compiled and provided by the Canadian Institute for Health Information (CIHI). However, the analyses, conclusions, opinions, and statements expressed in the material are those of the author(s), and not necessarily those of $\mathrm{ClHI}$.

Correspondence: Claire de Oliveira, PhD, Institute for Mental Health Policy Research, Centre for Addiction and Mental Health, 33 Russell Street, Room T414, Toronto, Ontario M5S 2S1, Canada. Email: claire.deoliveira@camh.ca

\section{References}

1. Yabroff KR, Lamont EB, Mariotto A, et al. Cost of care for elderly cancer patients in the United States. J Natl Cancer Inst 2008, 100:630-641.

2. de Oliveira C, Pataky R, Bremner KE, et al. Phase-specific and lifetime costs of cancer care in Ontario, Canada. BMC Cancer 2016;16:809.

3. de Oliveira C, Pataky R, Bremner KE, et al. Estimating the cost of cancer care in British Columbia and Ontario: a Canadian inter-provincial comparison. Healthc Policy 2017;12:95-108.

4. Canadian Cancer Statistics Advisory Committee. Canadian Cancer Statistics 2017. Available at: https://www.canada.ca/en/public-health/ services/chronic-diseases/cancer/canadian-cancer-statistics.html. Accessed August 1, 2019.

5. de Oliveira C, Weir S, Rangrej J, et al. The economic burden of cancer care in Canada: a population-based cost study. CMAJ Open 2018;6:E1-10.

6. Riley GF. Long-term trends in the concentration of Medicare spending. Health Aff (Millwood) 2007;26:808-816.

7. Smulowitz PB, Lipton R, Wharam JF, et al. Emergency department utilization after the implementation of Massachusetts health reform. Ann Emerg Med 2011;58:225-234.e1.

8. Ballard DW, Price M, Fung $\mathrm{V}$, et al. Validation of an algorithm for categorizing the severity of hospital emergency department visits. Med Care 2010;48:58-63.

9. Niefeld MR, Braunstein JB, Wu AW, et al. Preventable hospitalization among elderly Medicare beneficiaries with type 2 diabetes. Diabetes Care 2003:26:1344-1349.
10. Billings J, Anderson GM, Newman LS. Recent findings on preventable hospitalizations. Health Aff (Millwood) 1996;15:239-249.

11. Oster A, Bindman AB. Emergency department visits for ambulatory care sensitive conditions: insights into preventable hospitalizations. Med Care 2003;41:198-207.

12. Braunstein JB, Anderson GF, Gerstenblith G, et al. Noncardiac comorbidity increases preventable hospitalizations and mortality among Medicare beneficiaries with chronic heart failure. J Am Coll Cardiol 2003;42:1226-1233.

13. de Oliveira C, Cheng J, Kurdyak P. Determining preventable acute care spending among high-cost patients in a single-payer public health care system. Eur J Health Econ 2019;20:869-878.

14. Wodchis WP, Arthurs E, Khan Al, et al. Cost trajectories for cancer patients. Curr Oncol 2016;23(Suppl 1):S64-75.

15. Lam MB, Burke LG, Orav EJ, et al. Proportion of patients with cancer among high-cost Medicare beneficiaries: who they are and what drives their spending. Healthc (Amst) 2018;6:46-51

16. Joynt KE, Gawande AA, Orav EJ, et al. Contribution of preventable acute care spending to total spending for high-cost Medicare patients. JAMA 2013;309:2572-2578

17. Graven PF, Meath THA, Mendelson A, et al. Preventable acute care spending for high-cost patients across payer types. J Health Care Finance 2016;42:1-22.

18. Ronksley PE, Kobewka DM, McKay JA, et al. Clinical characteristics and preventable acute care spending among a high cost inpatient population. BMC Health Serv Res 2016;16:165. 
19. Wodchis WP, Bushmeneva K, Nikitovic M, McKillop I. Guidelines on person-level costing using administrative databases in Ontario. Available at: http://www.hsprn.ca/uploads/files/Guidelines_on_PersonLevel_ Costing_May_2013.pdf. Accessed August 1, 2019.

20. de Oliveira $\mathrm{C}$, Cheng J, Vigod S, et al. Patients with high mental health costs incur over 30 percent more costs than other high-cost patients. Health Aff (Millwood) 2016;35:36-43.

21. Wodchis WP, Austin PC, Henry DA. A 3-year study of high-cost users of health care. CMAJ 2016;188:182-188.

22. NYU Wagner. NYU ED algorithm: background. Available at: https://wagner. nyu.edu/faculty/billings/nyued-background. Accessed August 1, 2019.

23. Johnston KJ, Allen L, Melanson TA, et al. A "patch" to the NYU emergency department visit algorithm. Health Serv Res 2017;52:1264-1276.

24. Billings J, Parikh N, Mijanovich T. Emergency department use: the New York story. Issue Brief (Commonw Fund) 2000:1-12.

25. Agency for Healthcare Research and Quality. Prevention Quality Indicators overview. Available at: http://www.qualityindicators.ahrq.gov/ modules/pqi_overview.aspx. Accessed December 17, 2018.

26. Basu J, Friedman B, Burstin H. Primary care, HMO enrollment, and hospitalization for ambulatory care sensitive conditions: a new approach. Med Care 2002;40:1260-1269

27. Bindman AB, Grumbach K, Osmond D, et al. Preventable hospitalizations and access to health care. JAMA 1995;274:305-311.

28. Jiang HJ, Russo CA, Barrett ML. Nationwide frequency and costs of potentially preventable hospitalizations, 2006. HCUP statistical brief \#72. April 2009. US Agency for Healthcare Research and Quality, Rockville, MD. Available at: http://www.hcup-us.ahrq.gov/reports/statbriefs/ sb72.pdf. Accessed October 20, 2019.

29. Antoniou T, Zagorski B, Loutfy MR, et al. Validation of case-finding algorithms derived from administrative data for identifying adults living with human immunodeficiency virus infection. PLoS One 2011;6:e21748.

30. Benchimol El, Guttmann A, Mack DR, et al. Validation of international algorithms to identify adults with inflammatory bowel disease in health administrative data from Ontario, Canada. J Clin Epidemiol 2014;67:887-896.

31. Gershon AS, Wang C, Guan J, et al. Identifying patients with physiciandiagnosed asthma in health administrative databases. Can Respir J 2009;16:183-188.

32. Gershon AS, Wang C, Guan J, et al. Identifying individuals with physician-diagnosed COPD in health administrative databases. COPD 2009;6:388-394.

33. Hux JE, Ivis F, Flintoft $V$, et al. Diabetes in Ontario: determination of prevalence and incidence using a validated administrative data algorithm. Diabetes Care 2002;25:512-516.

34. Jaakkimainen RL, Bronskill SE, Tierney MC, et al. Identification of physician-diagnosed Alzheimer's disease and related dementias in population-based administrative data: a validation study using family physicians' electronic medical records. J Alzheimers Dis 2016:54 337-349.

35. Kurdyak $\mathrm{P}$, Lin $\mathrm{E}$, Green $\mathrm{D}$, et al. Validation of a population-based algorithm to detect chronic psychotic illness. Can J Psychiatry 2015;60: 362-368.

36. Moist LM, Fenton S, Kim JS, et al. Canadian Organ Replacement Registe (CORR): reflecting the past and embracing the future. Can J Kidney Health Dis 2014;1:26.

37. Prodhan S, King MJ, De P, et al. Health services data: the Ontario Cancer Registry (a unique, linked, and automated population-based registry). In: Sobolev B, Levy A, Goring S, eds. Data and Measures in Health Services Research.Boston, MA: Springer; 2016:1-27.

38. Schultz SE, Rothwell DM, Chen $Z$, et al. Identifying cases of congestive heart failure from administrative data: a validation study using primary care patient records. Chronic Dis Inj Can 2013;33:160-166.

39. Tu K, Chen Z, Lipscombe LL. Prevalence and incidence of hypertension from 1995 to 2005: a population-based study. CMAJ 2008;178: 1429-1435.

40. Widdifield J, Bernatsky S, Paterson JM, et al. Accuracy of Canadian health administrative databases in identifying patients with rheumatoid arthritis: a validation study using the medical records of rheumatologists. Arthritis Care Res (Hoboken) 2013;65:1582-1591.

41. Weiner JP, ed. The Johns Hopkins ACG Case-Mix System. Version 7.0 Release Notes. May, 2005. Baltimore, MD: Johns Hopkins Bloomberg School of Public Health; 2005.

42. Statistics Canada. The consumer price index. Ottawa (ON): Statistics Canada; 2016. Cat. no. 62-001-X.

43. de Oliveira $C$, Bremner KE, Pataky $R$, et al. Understanding the costs of cancer care before and after diagnosis for the 21 most common cancers in Ontario: a population-based descriptive study. CMAJ Open 2013;1:E1-8.

44. Laudicella M, Walsh B, Burns E, et al. Cost of care for cancer patients in England: evidence from population-based patient-level data. $\mathrm{Br} \mathrm{J}$ Cancer 2016;114:1286-1292.

45. Banegas MP, Yabroff KR, O'Keeffe-Rosetti MC, et al. Medical care costs associated with cancer in integrated delivery systems. J Natl Compr Canc Netw 2018;16:402-410.

46. de Oliveira C, Cheng J, Rehm J, et al. The role of mental health and addiction among high-cost patients: a population-based study. J Med Econ 2018;21:348-355.

47. Goyal RK, Wheeler SB, Kohler RE, et al. Health care utilization from chemotherapy-related adverse events among low-income breast cancer patients: effect of enrollment in a medical home program. N C Med J 2014;75:231-238.

48. Sparano JA, Gray RJ, Makower DF, et al. Adjuvant chemotherapy guided by a 21-gene expression assay in breast cancer. N Engl J Med 2018;379: $111-121$. 
Supplemental online content for:

\section{High-Cost Patients and Preventable Spending: A Population-Based Study}

Claire de Oliveira, PhD; Joyce Cheng, MSc; Kelvin Chan, MD, PhD, FRCPC; Craig C. Earle, MD, MSc, FRCPC; Murray Krahn, MD, MSc, FRCPC; and Nicole Mittmann, PhD

J Natl Compr Canc Netw 2020;18(1):23-31

eTable 1: Summary of Administrative Healthcare Databases

eTable 2: ICD-O Codes by Cancer Site

eAppendix 1: Agency for Healthcare Research and Quality Prevention Quality Indicators and Common Nonpreventable Clinical Diagnostic Groups 


\section{eTable 1. Summary of Administrative Healthcare Databases}

Database

Discharge Abstract Database (DAD)

Ontario Mental Health Reporting System (OMHRS)

Continuing Care Reporting System (CCRS)

National Rehabilitation Reporting System (NRS)

National Ambulatory Care Reporting System (NACRS)

Ontario Health Insurance Plan (OHIP) claims database

Outpatient and physician services

Acute inpatient hospitalizations

Rehabilitation and outpatient clinics

\section{Description}

Psychiatric inpatient hospitalizations

Complex continuing care, long-term care

Emergency department visits, day surgery,

Ontario Drug Benefit (ODB) program database

Outpatient prescription drugs dialysis). services).
A national database that contains demographic and clinical data on inpatient hospital admissions.

Collects demographic and clinical information on individuals admitted to designated adult psychiatric beds in Ontario. Some patients with mental health issues are still in inpatient acute beds (ie, the DAD).

Contains demographic and clinical information on individuals receiving facility-based

continuing care. Services include medical longterm care, rehabilitation, geriatric assessment, respite palliative care, and nursing home care.

Contains national data on rehabilitation facilities and clients, collected from participating adult inpatient rehabilitation facilities and programs.

Contains data for all ambulatory care including emergency department visits, day surgery, and outpatient clinic visits (eg, chemotherapy and

Covers all services and procedures provided by healthcare providers who can claim under OHIP (eg, physician and laboratory/diagnostic

Includes data on all drugs dispensed in community pharmacies and long-term care/ nursing facilities. Also covers prescription drugs listed in the provincial formulary for al seniors (aged $\geq 65$ years) and those aged $<65$ years on social assistance.

Home Care Database (HCD)

Home care
Provides data on government-funded services coordinated by Ontario's Community Care Access Centres for individuals requiring home care. 
eTable 2. ICD-O Codes by Cancer Site

Cancer

Head and neck

$\mathrm{CO9}{ }^{*}-\mathrm{C} 14^{*}$

Esophageal

Gastric

Colorectal

$\mathrm{C} 19.9$

Liver

Pancreatic

Lung

Leukemia

Melanoma

Female breast

Cervical

Uterine corpus

Ovarian

Prostate

Testicular

Kidney

Bladder

Brain

Thyroid

Lymphoma

Multiple myeloma

Other tumor sites

$\mathrm{C} 30^{*}-\mathrm{C} 33^{*}$

C15*

C16*

C20.9

C22.0

C25*

C34*

$\mathrm{C} 44^{*}$

$\mathrm{C} 53^{*}$

C54*

C55.9

C56.9

C61.9

C62*

C64.9

C65.9

C67*

$\mathrm{C} 71^{*}$

C73.9

C77*

$\mathrm{C} 07^{*}-\mathrm{CO} 8^{*}$
ICD-O Codes

C18.0, C18.2-C18.9

C42.0, C42.1, C42.4

C50* (female)

Not applicable; histology codes 9731, 9732, and 9734 were used to ascertain cancer site

C17*

C18.1

C21*

C22.1

C23.9

$\mathrm{C} 24$ *

C26*

C37.9

$\mathrm{C} 38^{*}-\mathrm{C} 41^{*}$

C42.2, C42.3

$\mathrm{C} 47^{*}-\mathrm{C} 49^{*}$

C50* (male)

C51*

C52.9

C57*

C58.9

C60*

C63*

C66*

C68*-C70*

C72*

C74*-C75*

C76*

C80.9 


\title{
eAppendix 1. Agency for Healthcare Research and Quality Prevention Quality Indicators and Common Nonpreventable Clinical Diagnostic Groups
}

\author{
Prevention Quality Indicators (PQIs) and ICD-10 Codes Used to Indicate Diagnosis \\ PQI 01: Diabetes Short-Term Complications
}

\begin{abstract}
Diabetes Short-Term Complications Diagnosis Codes: (ACDIASD)
E1010 Type 1 diabetes mellitus with ketoacidosis without coma

E1011 Type 1 diabetes mellitus with ketoacidosis with coma

E10641 Type 1 diabetes mellitus with hypoglycemia with coma

E1100 Type 2 diabetes mellitus with hyperosmolarity without nonketotichyperglycemic-hyperosmolar coma (NKHHC)

E1101 Type 2 diabetes mellitus with hyperosmolarity with coma

E11641 Type 2 diabetes mellitus with hypoglycemia with coma
\end{abstract}

PQI 02: Perforated Appendix

\section{Perforations or Abscesses of Appendix Diagnosis Codes: (ACSAPPD)}

K352 Acute appendicitis with generalized peritonitis

K353 Acute appendicitis with localized peritonitis

\section{PQI 03: Diabetes Long-Term Complications}

\section{Diabetes With Long-Term Complications Diagnosis Codes: (ACDIALD)}

E1021 Type 1 diabetes mellitus with diabetic nephropathy

E1022 Type 1 diabetes mellitus with diabetic chronic kidney disease

E1029 Type 1 diabetes mellitus with other diabetic kidney complication

E10311 Type 1 diabetes mellitus with unspecified diabetic retinopathy with macular edema

E10319 Type 1 diabetes mellitus with unspecified diabetic retinopathy without macular edema

E10321 Type 1 diabetes mellitus with mild nonproliferative diabetic retinopathy with macular edema

E103211 Type 1 diabetes mellitus with mild nonproliferative diabetic retinopathy with macular edema, right eye

E103212 Type 1 diabetes mellitus with mild nonproliferative diabetic retinopathy with macular edema, left eye

E103213 Type 1 diabetes mellitus with mild nonproliferative diabetic retinopathy with macular edema, bilateral

E103219 Type 1 diabetes mellitus with mild nonproliferative diabetic retinopathy with macular edema, unspecified eye

E10329 Type 1 diabetes mellitus with mild nonproliferative diabetic retinopathy without macular edema

E103291 Type 1 diabetes mellitus with mild nonproliferative diabetic retinopathy without macular edema, right eye

E103292 Type 1 diabetes mellitus with mild nonproliferative diabetic retinopathy without macular edema, left eye

E103293 Type 1 diabetes mellitus with mild nonproliferative diabetic retinopathy without macular edema, bilateral

E103299 Type 1 diabetes mellitus with mild nonproliferative diabetic retinopathy without macular edema, unspecified eye

E10331 Type 1 diabetes mellitus with moderate nonproliferative diabetic retinopathy with macular edema

E103311 Type 1 diabetes mellitus with moderate nonproliferative diabetic retinopathy with macular edema, right eye

E103312 Type 1 diabetes mellitus with moderate nonproliferative diabetic retinopathy with macular edema, left eye

E103313 Type 1 diabetes mellitus with moderate nonproliferative diabetic retinopathy with macular edema, bilateral

E103319 Type 1 diabetes mellitus with moderate nonproliferative diabetic retinopathy with macular edema, unspecified eye

E10339 Type 1 diabetes mellitus with moderate nonproliferative diabetic retinopathy without macular edema

E103391 Type 1 diabetes mellitus with moderate nonproliferative diabetic retinopathy without macular edema, right eye 
E103392 Type 1 diabetes mellitus with moderate nonproliferative diabetic retinopathy without macular edema, left eye E103393 Type 1 diabetes mellitus with moderate nonproliferative diabetic retinopathy without macular edema, bilateral E103399 Type 1 diabetes mellitus with moderate nonproliferative diabetic retinopathy without macular edema, unspecified eye E10341 Type 1 diabetes mellitus with severe nonproliferative diabetic retinopathy with macular edema

E103411 Type 1 diabetes mellitus with severe nonproliferative diabetic retinopathy with macular edema, right eye E103412 Type 1 diabetes mellitus with severe nonproliferative diabetic retinopathy with macular edema, left eye E103413 Type 1 diabetes mellitus with severe nonproliferative diabetic retinopathy with macular edema, bilateral E103419 Type 1 diabetes mellitus with severe nonproliferative diabetic retinopathy with macular edema, unspecified eye E10349 Type 1 diabetes mellitus with severe nonproliferative diabetic retinopathy without macular edema

E103491 Type 1 diabetes mellitus with severe nonproliferative diabetic retinopathy without macular edema, right eye E103492 Type 1 diabetes mellitus with severe nonproliferative diabetic retinopathy without macular edema, left eye E103493 Type 1 diabetes mellitus with severe nonproliferative diabetic retinopathy without macular edema, bilateral E103499 Type 1 diabetes mellitus with severe nonproliferative diabetic retinopathy without macular edema, unspecified eye E10351 Type 1 diabetes mellitus with proliferative diabetic retinopathy with macular edema

E103511 Type 1 diabetes mellitus with proliferative diabetic retinopathy with macular edema, right eye

E103512 Type 1 diabetes mellitus with proliferative diabetic retinopathy with macular edema, left eye

E103513 Type 1 diabetes mellitus with proliferative diabetic retinopathy with macular edema, bilateral

E103519 Type 1 diabetes mellitus with proliferative diabetic retinopathy with macular edema, unspecified eye

E103521 Type 1 diabetes mellitus with proliferative diabetic retinopathy with traction retinal detachment involving the macula, right eye

E103522 Type 1 diabetes mellitus with proliferative diabetic retinopathy with traction retinal detachment involving the macula, left eye

E103523 Type 1 diabetes mellitus with proliferative diabetic retinopathy with traction retinal detachment involving the macula, bilateral

E103529 Type 1 diabetes mellitus with proliferative diabetic retinopathy with traction retinal detachment involving the macula, unspecified eye

E103531 Type 1 diabetes mellitus with proliferative diabetic retinopathy with traction retinal detachment not involving the macula, right eye

E103532 Type 1 diabetes mellitus with proliferative diabetic retinopathy with traction retinal detachment not involving the macula, left eye

E103533 Type 1 diabetes mellitus with proliferative diabetic retinopathy with traction retinal detachment not involving the macula, bilateral

E103539 Type 1 diabetes mellitus with proliferative diabetic retinopathy with traction retinal detachment not involving the macula, unspecified eye

E103541 Type 1 diabetes mellitus with proliferative diabetic retinopathy with combined traction retinal detachment and rhegmatogenous retinal detachment, right eye

E103542 Type 1 diabetes mellitus with proliferative diabetic retinopathy with combined traction retinal detachment and rhegmatogenous retinal detachment, left eye

E103543 Type 1 diabetes mellitus with proliferative diabetic retinopathy with combined traction retinal detachment and rhegmatogenous retinal detachment, bilateral

E103549 Type 1 diabetes mellitus with proliferative diabetic retinopathy with combined traction retinal detachment and rhegmatogenous retinal detachment, unspecified eye

E103551 Type 1 diabetes mellitus with stable proliferative diabetic retinopathy, right eye

E103552 Type 1 diabetes mellitus with stable proliferative diabetic retinopathy, left eye

E103553 Type 1 diabetes mellitus with stable proliferative diabetic retinopathy, bilateral

E103559 Type 1 diabetes mellitus with stable proliferative diabetic retinopathy, unspecified eye

E10359 Type 1 diabetes mellitus with proliferative diabetic retinopathy without macular edema

E103591 Type 1 diabetes mellitus with proliferative diabetic retinopathy without macular edema, right eye

E103592 Type 1 diabetes mellitus with proliferative diabetic retinopathy without macular edema, left eye

E103593 Type 1 diabetes mellitus with proliferative diabetic retinopathy without macular edema, bilateral

(continued on next page) 
E103599 Type 1 diabetes mellitus with proliferative diabetic retinopathy without macular edema, unspecified eye E1036 Type 1 diabetes mellitus with diabetic cataract

E1037 $\times 1$ Type 1 diabetes mellitus with diabetic macular edema, resolved following treatment, right eye

E1037 $\times 2$ Type 1 diabetes mellitus with diabetic macular edema, resolved following treatment, left eye

E1037 $\times 3$ Type 1 diabetes mellitus with diabetic macular edema, resolved following treatment, bilateral

E1037 $\times 9$ Type 1 diabetes mellitus with diabetic macular edema, resolved following treatment, unspecified eye

E1039 Type 1 diabetes mellitus with other diabetic ophthalmic complication

E1040 Type 1 diabetes mellitus with diabetic neuropathy, unspecified

E1041 Type 1 diabetes mellitus with diabetic mononeuropathy

E1042 Type 1 diabetes mellitus with diabetic polyneuropathy

E1043 Type 1 diabetes mellitus with diabetic autonomic (poly)neuropathy

E1044 Type 1 diabetes mellitus with diabetic amyotrophy

E1049 Type 1 diabetes mellitus with other diabetic neurologic complication

E1051 Type 1 diabetes mellitus with diabetic peripheral angiopathy without gangrene

E1052 Type 1 diabetes mellitus with diabetic peripheral angiopathy with gangrene

E1059 Type 1 diabetes mellitus with other circulatory complications

E10610 Type 1 diabetes mellitus with diabetic neuropathic arthropathy

E10618 Type 1 diabetes mellitus with other diabetic arthropathy

E10620 Type 1 diabetes mellitus with diabetic dermatitis

E10621 Type 1 diabetes mellitus with foot ulcer

E10622 Type 1 diabetes mellitus with other skin ulcer

E10628 Type 1 diabetes mellitus with other skin complications

E10630 Type 1 diabetes mellitus with periodontal disease

E10638 Type 1 diabetes mellitus with other oral complications

E1069 Type 1 diabetes mellitus with other specified complication

E108 Type 1 diabetes mellitus with unspecified complications

E1121 Type 2 diabetes mellitus with diabetic nephropathy

E1122 Type 2 diabetes mellitus with diabetic chronic kidney disease

E1129 Type 2 diabetes mellitus with other diabetic kidney complication

E11311 Type 2 diabetes mellitus with unspecified diabetic retinopathy with macular edema

E11319 Type 2 diabetes mellitus with unspecified diabetic retinopathy without macular edema

E11321 Type 2 diabetes mellitus with mild nonproliferative diabetic retinopathy with macular edema

E113211 Type 2 diabetes mellitus with mild nonproliferative diabetic retinopathy with macular edema, right eye

E113212 Type 2 diabetes mellitus with mild nonproliferative diabetic retinopathy with macular edema, left eye

E113213 Type 2 diabetes mellitus with mild nonproliferative diabetic retinopathy with macular edema, bilateral

E113219 Type 2 diabetes mellitus with mild nonproliferative diabetic retinopathy with macular edema, unspecified eye

E11329 Type 2 diabetes mellitus with mild nonproliferative diabetic retinopathy without macular edema

E113291 Type 2 diabetes mellitus with mild nonproliferative diabetic retinopathy without macular edema, right eye

E113292 Type 2 diabetes mellitus with mild nonproliferative diabetic retinopathy without macular edema, left eye

E113293 Type 2 diabetes mellitus with mild nonproliferative diabetic retinopathy without macular edema, bilateral

E113299 Type 2 diabetes mellitus with mild nonproliferative diabetic retinopathy without macular edema, unspecified eye

E11331 Type 2 diabetes mellitus with moderate nonproliferative diabetic retinopathy with macular edema

E113311 Type 2 diabetes mellitus with moderate nonproliferative diabetic retinopathy with macular edema, right eye

E113312 Type 2 diabetes mellitus with moderate nonproliferative diabetic retinopathy with macular edema, left eye

E113313 Type 2 diabetes mellitus with moderate nonproliferative diabetic retinopathy with macular edema, bilateral

E113319 Type 2 diabetes mellitus with moderate nonproliferative diabetic retinopathy with macular edema, unspecified eye

E11339 Type 2 diabetes mellitus with moderate nonproliferative diabetic retinopathy without macular edema

(continued on next page) 
E113391 Type 2 diabetes mellitus with moderate nonproliferative diabetic retinopathy without macular edema, right eye E113392 Type 2 diabetes mellitus with moderate nonproliferative diabetic retinopathy without macular edema, left eye E113393 Type 2 diabetes mellitus with moderate nonproliferative diabetic retinopathy without macular edema, bilateral E113399 Type 2 diabetes mellitus with moderate nonproliferative diabetic retinopathy without macular edema, unspecified eye

E11341 Type 2 diabetes mellitus with severe nonproliferative diabetic retinopathy with macular edema

E113411 Type 2 diabetes mellitus with severe nonproliferative diabetic retinopathy with macular edema, right eye

E113412 Type 2 diabetes mellitus with severe nonproliferative diabetic retinopathy with macular edema, left eye

E113413 Type 2 diabetes mellitus with severe nonproliferative diabetic retinopathy with macular edema, bilateral E113419 Type 2 diabetes mellitus with severe nonproliferative diabetic retinopathy with macular edema, unspecified eye

E11349 Type 2 diabetes mellitus with severe nonproliferative diabetic retinopathy without macular edema

E113491 Type 2 diabetes mellitus with severe nonproliferative diabetic retinopathy without macular edema, right eye

E113492 Type 2 diabetes mellitus with severe nonproliferative diabetic retinopathy without macular edema, left eye

E113493 Type 2 diabetes mellitus with severe nonproliferative diabetic retinopathy without macular edema, bilateral

E113499 Type 2 diabetes mellitus with severe nonproliferative diabetic retinopathy without macular edema, unspecified eye

E11351 Type 2 diabetes mellitus with proliferative diabetic retinopathy with macular edema

E113511 Type 2 diabetes mellitus with proliferative diabetic retinopathy with macular edema, right eye

E113512 Type 2 diabetes mellitus with proliferative diabetic retinopathy with macular edema, left eye

E113513 Type 2 diabetes mellitus with proliferative diabetic retinopathy with macular edema, bilateral

E113519 Type 2 diabetes mellitus with proliferative diabetic retinopathy with macular edema, unspecified eye

E113521 Type 2 diabetes mellitus with proliferative diabetic retinopathy with traction retinal detachment involving the macula, right eye

E113522 Type 2 diabetes mellitus with proliferative diabetic retinopathy with traction retinal detachment involving the macula, left eye

E113523 Type 2 diabetes mellitus with proliferative diabetic retinopathy with traction retinal detachment involving the macula, bilateral

E113529 Type 2 diabetes mellitus with proliferative diabetic retinopathy with traction retinal detachment involving the macula, unspecified eye

E113531 Type 2 diabetes mellitus with proliferative diabetic retinopathy with traction retinal detachment not involving the macula, right eye

E113532 Type 2 diabetes mellitus with proliferative diabetic retinopathy with traction retinal detachment not involving the macula, left eye

E113533 Type 2 diabetes mellitus with proliferative diabetic retinopathy with traction retinal detachment not involving the macula, bilateral

E113539 Type 2 diabetes mellitus with proliferative diabetic retinopathy with traction retinal detachment not involving the macula, unspecified eye

E113541 Type 2 diabetes mellitus with proliferative diabetic retinopathy with combined traction retinal detachment and rhegmatogenous retinal detachment, right eye

E113542 Type 2 diabetes mellitus with proliferative diabetic retinopathy with combined traction retinal detachment and rhegmatogenous retinal detachment, left eye

E113543 Type 2 diabetes mellitus with proliferative diabetic retinopathy with combined traction retinal detachment and rhegmatogenous retinal detachment, bilateral

E113549 Type 2 diabetes mellitus with proliferative diabetic retinopathy with combined traction retinal detachment and rhegmatogenous retinal detachment, unspecified eye

E113551 Type 2 diabetes mellitus with stable proliferative diabetic retinopathy, right eye

E113552 Type 2 diabetes mellitus with stable proliferative diabetic retinopathy, left eye

E113553 Type 2 diabetes mellitus with stable proliferative diabetic retinopathy, bilateral

E113559 Type 2 diabetes mellitus with stable proliferative diabetic retinopathy, unspecified eye

E11359 Type 2 diabetes mellitus with proliferative diabetic retinopathy without macular edema

E113591 Type 2 diabetes mellitus with proliferative diabetic retinopathy without macular edema, right eye

(continued on next page) 
E113592 Type 2 diabetes mellitus with proliferative diabetic retinopathy without macular edema, left eye E113593 Type 2 diabetes mellitus with proliferative diabetic retinopathy without macular edema, bilateral E113599 Type 2 diabetes mellitus with proliferative diabetic retinopathy without macular edema, unspecified eye E1136 Type 2 diabetes mellitus with diabetic cataract

E1137 $\times 1$ Type 2 diabetes mellitus with diabetic macular edema, resolved following treatment, right eye

E1137 $\times 2$ Type 2 diabetes mellitus with diabetic macular edema, resolved following treatment, left eye

E1137 $\times 3$ Type 2 diabetes mellitus with diabetic macular edema, resolved following treatment, bilateral

E1137 $\times 9$ Type 2 diabetes mellitus with diabetic macular edema, resolved following treatment, unspecified eye

E1139 Type 2 diabetes mellitus with other diabetic ophthalmic complication

E1140 Type 2 diabetes mellitus with diabetic neuropathy, unspecified

E1141 Type 2 diabetes mellitus with diabetic mononeuropathy

E1142 Type 2 diabetes mellitus with diabetic polyneuropathy

E1143 Type 2 diabetes mellitus with diabetic autonomic (poly)neuropathy

E1144 Type 2 diabetes mellitus with diabetic amyotrophy

E1149 Type 2 diabetes mellitus with other diabetic neurologic complication

E1151 Type 2 diabetes mellitus with diabetic peripheral angiopathy without gangrene

E1152 Type 2 diabetes mellitus with diabetic peripheral angiopathy with gangrene

E1159 Type 2 diabetes mellitus with other circulatory complications

E11610 Type 2 diabetes mellitus with diabetic neuropathic arthropathy

E11618 Type 2 diabetes mellitus with other diabetic arthropathy

E11620 Type 2 diabetes mellitus with diabetic dermatitis

E11621 Type 2 diabetes mellitus with foot ulcer

E11622 Type 2 diabetes mellitus with other skin ulcer

E11628 Type 2 diabetes mellitus with other skin complications

E11630 Type 2 diabetes mellitus with periodontal disease

E11638 Type 2 diabetes mellitus with other oral complications

E1169 Type 2 diabetes mellitus with other specified complication

E118 Type 2 diabetes mellitus with unspecified complications

PQI 05: Chronic Obstructive Pulmonary Disease (COPD) or Asthma in Older Adults

\section{COPD (Excluding Acute Bronchitis) Diagnosis Codes: (ACCOPDD)}

J410 Simple chronic bronchitis

J411 Mucopurulent chronic bronchitis

J418 Mixed simple and mucopurulent chronic bronchitis

J42 Unspecified chronic bronchitis

J430 Unilateral pulmonary emphysema (MacLeod's syndrome)

J431 Panlobular emphysema

J432 Centrilobular emphysema

J439 Emphysema, unspecified

J440 COPD with acute lower respiratory infection

J441 COPD with (acute) exacerbation

J449 COPD, unspecified

J470 Bronchiectasis with acute lower respiratory infection

J471 Bronchiectasis with (acute) exacerbation

J479 Bronchiectasis, uncomplicated 


\section{PQI 07: Hypertension}

\section{Hypertension Diagnosis Codes: (ACSHYPD)}

I10 Essential (primary) hypertension

I119 Hypertensive heart disease without heart failure

I129 Hypertensive chronic kidney disease with stage I through stage IV chronic kidney disease, or unspecified chronic kidney disease I1310 Hypertensive heart and chronic kidney disease without heart failure, with stage I through stage IV chronic kidney disease, or unspecified chronic kidney disease

I160 Hypertensive urgency

I161 Hypertensive emergency

I169 Hypertensive crisis, unspecified

PQI 08: Heart Failure

\section{Heart Failure Diagnosis Codes: (MRTCHFD)}

I0981 Rheumatic heart failure

I110 Hypertensive heart disease with heart failure

I130 Hypertensive heart and chronic kidney disease with heart failure and stage I through stage IV chronic kidney disease, or unspecified chronic kidney disease

I132 Hypertensive heart and chronic kidney disease with heart failure and with stage V chronic kidney disease, or end-stage renal disease I501 Left ventricular failure

I5020 Unspecified systolic (congestive) heart failure

I5021 Acute systolic (congestive) heart failure

I5022 Chronic systolic (congestive) heart failure

I5023 Acute on chronic systolic (congestive) heart failure

I5030 Unspecified diastolic (congestive) heart failure

I5031 Acute diastolic (congestive) heart failure

I5032 Chronic diastolic (congestive) heart failure

I5033 Acute on chronic diastolic (congestive) heart failure

I5040 Unspecified combined systolic (congestive) and diastolic (congestive) heart failure

I5041 Acute combined systolic (congestive) and diastolic (congestive) heart failure

I5042 Chronic combined systolic (congestive) and diastolic (congestive) heart failure

I5043 Acute on chronic combined systolic (congestive) and diastolic (congestive) heart failure

I509 Heart failure, unspecified

PQI 09: Low Birth Weight

Not included

PQI 10: Dehydration

\section{Dehydration Diagnosis Codes: (ACSDEHD)}

E860 Dehydration

E861 Hypovolemia

E869 Volume depletion, unspecified

\section{PQI 11: Community-Acquired Pneumonia}

\section{Community-Acquired Bacterial Pneumonia Diagnosis Codes: (ACSBACD)}

J13 Pneumonia due to Streptococcus pneumoniae

J14 Pneumonia due to Haemophilus influenzae 
J15211 Pneumonia due to methicillin-susceptible Staphylococcus aureus

J15212 Pneumonia due to methicillin-resistant Staphylococcus aureus

J153 Pneumonia due to Streptococcus, group B

J154 Pneumonia due to other streptococci

J157 Pneumonia due to Mycoplasma pneumoniae

J159 Unspecified bacterial pneumonia

J160 Chlamydial pneumonia

J168 Pneumonia due to other specified infectious organisms

J180 Bronchopneumonia, unspecified organism

J181 Lobar pneumonia, unspecified organism

J188 Other pneumonia, unspecified organism

J189 Pneumonia, unspecified organism

PQI 12: Urinary Tract Infection

Urinary Tract Infection Diagnosis Codes: (ACSUTID)

N10 Acute tubulo-interstitial nephritis

N119 Chronic tubulo-interstitial nephritis, unspecified

N12 Tubulo-interstitial nephritis, not specified as acute or chronic

N151 Renal and perinephric abscess

N159 Renal tubulo-interstitial disease, unspecified

N16 Renal tubulo-interstitial disorders in diseases classified elsewhere

N2884 Pyelitis cystica

N2885 Pyeloureteritis cystica

N2886 Ureteritis cystica

N3000 Acute cystitis without hematuria

N3001 Acute cystitis with hematuria

N3090 Cystitis, unspecified without hematuria

N3091 Cystitis, unspecified with hematuria

N390 Urinary tract infection, site not specified

\section{PQI 14: Uncontrolled Diabetes}

Uncontrolled Diabetes Without Mention of a Short-Term or Long-Term Complication Diagnosis Codes: (ACDIAUD)

E10649 Type 1 diabetes mellitus with hypoglycemia without coma

E1065 Type 1 diabetes mellitus with hyperglycemia

E11649 Type 2 diabetes mellitus with hypoglycemia without coma

E1165 Type 2 diabetes mellitus with hyperglycemia

PQI 15: Asthma in Younger Adults

Asthma Diagnosis Codes: (ACSASTD)

J4521 Mild intermittent asthma with (acute) exacerbation

J4522 Mild intermittent asthma with status asthmaticus

J4531 Mild persistent asthma with (acute) exacerbation

J4532 Mild persistent asthma with status asthmaticus 
J4541 Moderate persistent asthma with (acute) exacerbation

J4542 Moderate persistent asthma with status asthmaticus

J4551 Severe persistent asthma with (acute) exacerbation

J4552 Severe persistent asthma with status asthmaticus

J45901 Unspecified asthma with (acute) exacerbation

J45902 Unspecified asthma with status asthmaticus

J45990 Exercise-induced bronchospasm

J45991 Cough-variant asthma

J45998 Other asthma

\section{POI 16: Lower-Extremity Amputation Among Patients With Diabetes}

\section{Lower-Extremity Amputation Procedure Codes: (ACSLEAP)}

Note: These are Canadian Classification of Health Intervention (CCI) codes, which correspond to the ICD-10 Procedure Coding System codes for this PQI; more information on CCI codes can be found at https://www.cihi.calen/cci-codingstructure.

1VA93 Amputation, hip joint

1VC93 Amputation, femur

1VG93 Amputation, knee joint

1VQ93 Amputation, tibia and fibula

1WA93 Amputation, ankle joint

1WE93 Amputation, tarsal bones and intertarsal joints (hindfoot, midfoot)

1WI93 Amputation, first metatarsal bone and first metatarsophalangeal joint

1WJ93 Amputation, tarsometatarsal joints, metatarsal bones, and metatarsophalangeal joints (forefoot)

1WK93 Amputation, first phalanx of foot

1WL93 Amputation, phalanx of foot

\section{Traumatic Amputation of the Lower Extremity Diagnosis Codes: (ACLEA2D)}

Note: These codes were excluded in line with PQI 16 Lower-Extremity Amputation Among Patients With Diabetes Rate (ie, codes with a main diagnosis of S78*, S88*, and S98*). See http://www.qualityindicators.ahrq.gov/Downloads/Modules/PQI/ V70/TechSpecs/PQI_16_Lower-Extremity_Amputation_among_Patients_with_Diabetes_Rate.pdf

S78011A Complete traumatic amputation at right hip joint, initial encounter

S78012A Complete traumatic amputation at left hip joint, initial encounter

S78019A Complete traumatic amputation at unspecified hip joint, initial encounter

S78021A Partial traumatic amputation at right hip joint, initial encounter

S78022A Partial traumatic amputation at left hip joint, initial encounter

S78029A Partial traumatic amputation at unspecified hip joint, initial encounter

S78111A Complete traumatic amputation at level between right hip and knee, initial encounter

S78112A Complete traumatic amputation at level between left hip and knee, initial encounter

S78119A Complete traumatic amputation at level between unspecified hip and knee, initial encounter

S78121A Partial traumatic amputation at level between right hip and knee, initial encounter

S78122A Partial traumatic amputation at level between left hip and knee, initial encounter

S78129A Partial traumatic amputation at level between unspecified hip and knee, initial encounter

S78911A Complete traumatic amputation of right hip and thigh, level unspecified, initial encounter

S78912A Complete traumatic amputation of left hip and thigh, level unspecified, initial encounter

S78919A Complete traumatic amputation of unspecified hip and thigh, level unspecified, initial encounter

S78921A Partial traumatic amputation of right hip and thigh, level unspecified, initial encounter 
S78922A Partial traumatic amputation of left hip and thigh, level unspecified, initial encounter S78929A Partial traumatic amputation of unspecified hip and thigh, level unspecified, initial encounter S88011A Complete traumatic amputation at knee level, right lower leg, initial encounter S88012A Complete traumatic amputation at knee level, left lower leg, initial encounter S88019A Complete traumatic amputation at knee level, unspecified lower leg, initial encounter S88021A Partial traumatic amputation at knee level, right lower leg, initial encounter S88022A Partial traumatic amputation at knee level, left lower leg, initial encounter S88029A Partial traumatic amputation at knee level, unspecified lower leg, initial encounter S88111A Complete traumatic amputation at level between knee and ankle, right lower leg, initial encounter S88112A Complete traumatic amputation at level between knee and ankle, left lower leg, initial encounter S88119A Complete traumatic amputation at level between knee and ankle, unspecified lower leg, initial encounter S88121A Partial traumatic amputation at level between knee and ankle, right lower leg, initial encounter S88122A Partial traumatic amputation at level between knee and ankle, left lower leg, initial encounter S88129A Partial traumatic amputation at level between knee and ankle, unspecified lower leg, initial encounter S88911A Complete traumatic amputation of right lower leg, level unspecified, initial encounter S88912A Complete traumatic amputation of left lower leg, level unspecified, initial encounter S88919A Complete traumatic amputation of unspecified lower leg, level unspecified, initial encounter S88921A Partial traumatic amputation of right lower leg, level unspecified, initial encounter S88922A Partial traumatic amputation of left lower leg, level unspecified, initial encounter S88929A Partial traumatic amputation of unspecified lower leg, level unspecified, initial encounter S98012A Complete traumatic amputation of left foot at ankle level, initial encounter S98019A Complete traumatic amputation of unspecified foot at ankle level, initial encounter S98021A Partial traumatic amputation of right foot at ankle level, initial encounter S98022A Partial traumatic amputation of left foot at ankle level, initial encounter S98029A Partial traumatic amputation of unspecified foot at ankle level, initial encounter S98111A Complete traumatic amputation of right great toe, initial encounter S98112A Complete traumatic amputation of left great toe, initial encounter S98119A Complete traumatic amputation of unspecified great toe, initial encounter S98121A Partial traumatic amputation of right great toe, initial encounter S98122A Partial traumatic amputation of left great toe, initial encounter S98129A Partial traumatic amputation of unspecified great toe, initial encounter S98131A Complete traumatic amputation of one right lesser toe, initial encounter S98132A Complete traumatic amputation of one left lesser toe, initial encounter S98139A Complete traumatic amputation of one unspecified lesser toe, initial encounter S98141A Partial traumatic amputation of one right lesser toe, initial encounter S98142A Partial traumatic amputation of one left lesser toe, initial encounter S98149A Partial traumatic amputation of one unspecified lesser toe, initial encounter S98211A Complete traumatic amputation of two or more right lesser toes, initial encounter S98212A Complete traumatic amputation of two or more left lesser toes, initial encounter S98219A Complete traumatic amputation of two or more unspecified lesser toes, initial encounter S98221A Partial traumatic amputation of two or more right lesser toes, initial encounter S98222A Partial traumatic amputation of two or more left lesser toes, initial encounter S98229A Partial traumatic amputation of two or more unspecified lesser toes, initial encounter S98311A Complete traumatic amputation of right midfoot, initial encounter S98312A Complete traumatic amputation of left midfoot, initial encounter S98319A Complete traumatic amputation of unspecified midfoot, initial encounter 
S98321A Partial traumatic amputation of right midfoot, initial encounter S98322A Partial traumatic amputation of left midfoot, initial encounter S98329A Partial traumatic amputation of unspecified midfoot, initial encounter

S98911A Complete traumatic amputation of right foot, level unspecified, initial encounter S98912A Complete traumatic amputation of left foot, level unspecified, initial encounter S98919A Complete traumatic amputation of unspecified foot, level unspecified, initial encounter S98921A Partial traumatic amputation of right foot, level unspecified, initial encounter S98922A Partial traumatic amputation of left foot, level unspecified, initial encounter S98929A Partial traumatic amputation of unspecified foot, level unspecified, initial encounter

\section{Diabetes Diagnosis Codes: (ACSLEAD)}

E1010 Type 1 diabetes mellitus with ketoacidosis without coma

E1011 Type 1 diabetes mellitus with ketoacidosis with coma

E1021 Type 1 diabetes mellitus with diabetic nephropathy

E1022 Type 1 diabetes mellitus with diabetic chronic kidney disease

E1029 Type 1 diabetes mellitus with other diabetic kidney complication

E10311 Type 1 diabetes mellitus with unspecified diabetic retinopathy with macular edema

E10319 Type 1 diabetes mellitus with unspecified diabetic retinopathy without macular edema

E10321 Type 1 diabetes mellitus with mild nonproliferative diabetic retinopathy with macular edema

E103211 Type 1 diabetes mellitus with mild nonproliferative diabetic retinopathy with macular edema, right eye

E103212 Type 1 diabetes mellitus with mild nonproliferative diabetic retinopathy with macular edema, left eye

E103213 Type 1 diabetes mellitus with mild nonproliferative diabetic retinopathy with macular edema, bilateral

E103219 Type 1 diabetes mellitus with mild nonproliferative diabetic retinopathy with macular edema, unspecified eye

E10329 Type 1 diabetes mellitus with mild nonproliferative diabetic retinopathy without macular edema

E103291 Type 1 diabetes mellitus with mild nonproliferative diabetic retinopathy without macular edema, right eye

E103292 Type 1 diabetes mellitus with mild nonproliferative diabetic retinopathy without macular edema, left eye

E103293 Type 1 diabetes mellitus with mild nonproliferative diabetic retinopathy without macular edema, bilateral

E103299 Type 1 diabetes mellitus with mild nonproliferative diabetic retinopathy without macular edema, unspecified eye

E10331 Type 1 diabetes mellitus with moderate nonproliferative diabetic retinopathy with macular edema

E103311 Type 1 diabetes mellitus with moderate nonproliferative diabetic retinopathy with macular edema, right eye

E103312 Type 1 diabetes mellitus with moderate nonproliferative diabetic retinopathy with macular edema, left eye

E103313 Type 1 diabetes mellitus with moderate nonproliferative diabetic retinopathy with macular edema, bilateral

E103319 Type 1 diabetes mellitus with moderate nonproliferative diabetic retinopathy with macular edema, unspecified eye

E10339 Type 1 diabetes mellitus with moderate nonproliferative diabetic retinopathy without macular edema

E103391 Type 1 diabetes mellitus with moderate nonproliferative diabetic retinopathy without macular edema, right eye

E103392 Type 1 diabetes mellitus with moderate nonproliferative diabetic retinopathy without macular edema, left eye

E103393 Type 1 diabetes mellitus with moderate nonproliferative diabetic retinopathy without macular edema, bilateral

E10341 Type 1 diabetes mellitus with severe nonproliferative diabetic retinopathy with macular edema

E103399 Type 1 diabetes mellitus with moderate nonproliferative diabetic retinopathy without macular edema, unspecified eye

E103411 Type 1 diabetes mellitus with severe nonproliferative diabetic retinopathy with macular edema, right eye

E103412 Type 1 diabetes mellitus with severe nonproliferative diabetic retinopathy with macular edema, left eye

E103413 Type 1 diabetes mellitus with severe nonproliferative diabetic retinopathy with macular edema, bilateral

E103419 Type 1 diabetes mellitus with severe nonproliferative diabetic retinopathy with macular edema, unspecified eye

E10349 Type 1 diabetes mellitus with severe nonproliferative diabetic retinopathy without macular edema

E103491 Type 1 diabetes mellitus with severe nonproliferative diabetic retinopathy without macular edema, right eye

E103492 Type 1 diabetes mellitus with severe nonproliferative diabetic retinopathy without macular edema, left eye 
E103493 Type 1 diabetes mellitus with severe nonproliferative diabetic retinopathy without macular edema, bilateral E103499 Type 1 diabetes mellitus with severe nonproliferative diabetic retinopathy without macular edema, unspecified eye E10351 Type 1 diabetes mellitus with proliferative diabetic retinopathy with macular edema

E103511 Type 1 diabetes mellitus with proliferative diabetic retinopathy with macular edema, right eye

E103512 Type 1 diabetes mellitus with proliferative diabetic retinopathy with macular edema, left eye

E103513 Type 1 diabetes mellitus with proliferative diabetic retinopathy with macular edema, bilateral

E103519 Type 1 diabetes mellitus with proliferative diabetic retinopathy with macular edema, unspecified eye

E103521 Type 1 diabetes mellitus with proliferative diabetic retinopathy with traction retinal detachment involving the macula, right eye

E103522 Type 1 diabetes mellitus with proliferative diabetic retinopathy with traction retinal detachment involving the macula, left eye

E103523 Type 1 diabetes mellitus with proliferative diabetic retinopathy with traction retinal detachment involving the macula, bilateral

E103529 Type 1 diabetes mellitus with proliferative diabetic retinopathy with traction retinal detachment involving the macula, unspecified eye

E103531 Type 1 diabetes mellitus with proliferative diabetic retinopathy with traction retinal detachment not involving the macula, right eye

E103532 Type 1 diabetes mellitus with proliferative diabetic retinopathy with traction retinal detachment not involving the macula, left eye

E103533 Type 1 diabetes mellitus with proliferative diabetic retinopathy with traction retinal detachment not involving the macula, bilateral

E103539 Type 1 diabetes mellitus with proliferative diabetic retinopathy with traction retinal detachment not involving the macula, unspecified eye

E103541 Type 1 diabetes mellitus with proliferative diabetic retinopathy with combined traction retinal detachment and rhegmatogenous retinal detachment, right eye

E103542 Type 1 diabetes mellitus with proliferative diabetic retinopathy with combined traction retinal detachment and rhegmatogenous retinal detachment, left eye

E103543 Type 1 diabetes mellitus with proliferative diabetic retinopathy with combined traction retinal detachment and rhegmatogenous retinal detachment, bilateral

E103549 Type 1 diabetes mellitus with proliferative diabetic retinopathy with combined traction retinal detachment and rhegmatogenous retinal detachment, unspecified eye

E103551 Type 1 diabetes mellitus with stable proliferative diabetic retinopathy, right eye

E103552 Type 1 diabetes mellitus with stable proliferative diabetic retinopathy, left eye

E103553 Type 1 diabetes mellitus with stable proliferative diabetic retinopathy, bilateral

E103559 Type 1 diabetes mellitus with stable proliferative diabetic retinopathy, unspecified eye

E10359 Type 1 diabetes mellitus with proliferative diabetic retinopathy without macular edema

E103591 Type 1 diabetes mellitus with proliferative diabetic retinopathy without macular edema, right eye

E103592 Type 1 diabetes mellitus with proliferative diabetic retinopathy without macular edema, left eye

E103593 Type 1 diabetes mellitus with proliferative diabetic retinopathy without macular edema, bilateral

E103599 Type 1 diabetes mellitus with proliferative diabetic retinopathy without macular edema, unspecified eye

E1036 Type 1 diabetes mellitus with diabetic cataract

E1037 $\times 1$ Type 1 diabetes mellitus with diabetic macular edema, resolved following treatment, right eye

E1037 $\times 2$ Type 1 diabetes mellitus with diabetic macular edema, resolved following treatment, left eye

E1037 $\times 3$ Type 1 diabetes mellitus with diabetic macular edema, resolved following treatment, bilateral

E1037 $\times 9$ Type 1 diabetes mellitus with diabetic macular edema, resolved following treatment, unspecified eye

E1039 Type 1 diabetes mellitus with other diabetic ophthalmic complication

E1040 Type 1 diabetes mellitus with diabetic neuropathy, unspecified

E1041 Type 1 diabetes mellitus with diabetic mononeuropathy

E1042 Type 1 diabetes mellitus with diabetic polyneuropathy

E1043 Type 1 diabetes mellitus with diabetic autonomic (poly)neuropathy

(continued on next page) 
E1044 Type 1 diabetes mellitus with diabetic amyotrophy

E1049 Type 1 diabetes mellitus with other diabetic neurologic complication

E1051 Type 1 diabetes mellitus with diabetic peripheral angiopathy without gangrene

E1052 Type 1 diabetes mellitus with diabetic peripheral angiopathy with gangrene

E1059 Type 1 diabetes mellitus with other circulatory complications

E10610 Type 1 diabetes mellitus with diabetic neuropathic arthropathy

E10618 Type 1 diabetes mellitus with other diabetic arthropathy

E10620 Type 1 diabetes mellitus with diabetic dermatitis

E10621 Type 1 diabetes mellitus with foot ulcer

E10622 Type 1 diabetes mellitus with other skin ulcer

E10628 Type 1 diabetes mellitus with other skin complications

E10630 Type 1 diabetes mellitus with periodontal disease

E10638 Type 1 diabetes mellitus with other oral complications

E10641 Type 1 diabetes mellitus with hypoglycemia with coma

E10649 Type 1 diabetes mellitus with hypoglycemia without coma

E1065 Type 1 diabetes mellitus with hyperglycemia

E1069 Type 1 diabetes mellitus with other specified complication

E108 Type 1 diabetes mellitus with unspecified complications

E109 Type 1 diabetes mellitus without complications

E1100 Type 2 diabetes mellitus with hyperosmolarity without nonketotic hyperglycemic-hyperosmolar coma (NKHHC)

E1101 Type 2 diabetes mellitus with hyperosmolarity with coma

E1121 Type 2 diabetes mellitus with diabetic nephropathy

E1122 Type 2 diabetes mellitus with diabetic chronic kidney disease

E1129 Type 2 diabetes mellitus with other diabetic kidney complication

E11311 Type 2 diabetes mellitus with unspecified diabetic retinopathy with macular edema

E11319 Type 2 diabetes mellitus with unspecified diabetic retinopathy without macular edema

E11321 Type 2 diabetes mellitus with mild nonproliferative diabetic retinopathy with macular edema

E113211 Type 2 diabetes mellitus with mild nonproliferative diabetic retinopathy with macular edema, right eye

E113212 Type 2 diabetes mellitus with mild nonproliferative diabetic retinopathy with macular edema, left eye

E113213 Type 2 diabetes mellitus with mild nonproliferative diabetic retinopathy with macular edema, bilateral

E113219 Type 2 diabetes mellitus with mild nonproliferative diabetic retinopathy with macular edema, unspecified eye

E11329 Type 2 diabetes mellitus with mild nonproliferative diabetic retinopathy without macular edema

E113291 Type 2 diabetes mellitus with mild nonproliferative diabetic retinopathy without macular edema, right eye

E113292 Type 2 diabetes mellitus with mild nonproliferative diabetic retinopathy without macular edema, left eye

E113293 Type 2 diabetes mellitus with mild nonproliferative diabetic retinopathy without macular edema, bilateral

E113299 Type 2 diabetes mellitus with mild nonproliferative diabetic retinopathy without macular edema, unspecified eye

E11331 Type 2 diabetes mellitus with moderate nonproliferative diabetic retinopathy with macular edema

E113311 Type 2 diabetes mellitus with moderate nonproliferative diabetic retinopathy with macular edema, right eye

E113312 Type 2 diabetes mellitus with moderate nonproliferative diabetic retinopathy with macular edema, left eye

E113313 Type 2 diabetes mellitus with moderate nonproliferative diabetic retinopathy with macular edema, bilateral

E113319 Type 2 diabetes mellitus with moderate nonproliferative diabetic retinopathy with macular edema, unspecified eye

E11339 Type 2 diabetes mellitus with moderate nonproliferative diabetic retinopathy without macular edema

E113391 Type 2 diabetes mellitus with moderate nonproliferative diabetic retinopathy without macular edema, right eye

E113392 Type 2 diabetes mellitus with moderate nonproliferative diabetic retinopathy without macular edema, left eye

E113393 Type 2 diabetes mellitus with moderate nonproliferative diabetic retinopathy without macular edema, bilateral

(continued on next page) 
E113399 Type 2 diabetes mellitus with moderate nonproliferative diabetic retinopathy without macular edema, unspecified eye

E11341 Type 2 diabetes mellitus with severe nonproliferative diabetic retinopathy with macular edema

E113411 Type 2 diabetes mellitus with severe nonproliferative diabetic retinopathy with macular edema, right eye

E113412 Type 2 diabetes mellitus with severe nonproliferative diabetic retinopathy with macular edema, left eye

E113413 Type 2 diabetes mellitus with severe nonproliferative diabetic retinopathy with macular edema, bilateral

E113419 Type 2 diabetes mellitus with severe nonproliferative diabetic retinopathy with macular edema, unspecified eye

E11349 Type 2 diabetes mellitus with severe nonproliferative diabetic retinopathy without macular edema

E113491 Type 2 diabetes mellitus with severe nonproliferative diabetic retinopathy without macular edema, right eye

E113492 Type 2 diabetes mellitus with severe nonproliferative diabetic retinopathy without macular edema, left eye

E113493 Type 2 diabetes mellitus with severe nonproliferative diabetic retinopathy without macular edema, bilateral

E113499 Type 2 diabetes mellitus with severe nonproliferative diabetic retinopathy without macular edema, unspecified eye

E11351 Type 2 diabetes mellitus with proliferative diabetic retinopathy with macular edema

E113511 Type 2 diabetes mellitus with proliferative diabetic retinopathy with macular edema, right eye

E113512 Type 2 diabetes mellitus with proliferative diabetic retinopathy with macular edema, left eye

E113513 Type 2 diabetes mellitus with proliferative diabetic retinopathy with macular edema, bilateral

E113519 Type 2 diabetes mellitus with proliferative diabetic retinopathy with macular edema, unspecified eye

E113521 Type 2 diabetes mellitus with proliferative diabetic retinopathy with traction retinal detachment involving the macula, right eye

E113522 Type 2 diabetes mellitus with proliferative diabetic retinopathy with traction retinal detachment involving the macula, left eye

E113523 Type 2 diabetes mellitus with proliferative diabetic retinopathy with traction retinal detachment involving the macula, bilateral

E113529 Type 2 diabetes mellitus with proliferative diabetic retinopathy with traction retinal detachment involving the macula, unspecified eye

E113531 Type 2 diabetes mellitus with proliferative diabetic retinopathy with traction retinal detachment not involving the macula, right eye

E113532 Type 2 diabetes mellitus with proliferative diabetic retinopathy with traction retinal detachment not involving the macula, left eye

E113533 Type 2 diabetes mellitus with proliferative diabetic retinopathy with traction retinal detachment not involving the macula, bilateral

E113539 Type 2 diabetes mellitus with proliferative diabetic retinopathy with traction retinal detachment not involving the macula, unspecified eye

E113541 Type 2 diabetes mellitus with proliferative diabetic retinopathy with combined traction retinal detachment and rhegmatogenous retinal detachment, right eye

E113542 Type 2 diabetes mellitus with proliferative diabetic retinopathy with combined traction retinal detachment and rhegmatogenous retinal detachment, left eye

E113543 Type 2 diabetes mellitus with proliferative diabetic retinopathy with combined traction retinal detachment and rhegmatogenous retinal detachment, bilateral

E113549 Type 2 diabetes mellitus with proliferative diabetic retinopathy with combined traction retinal detachment and rhegmatogenous retinal detachment, unspecified eye

E113551 Type 2 diabetes mellitus with stable proliferative diabetic retinopathy, right eye

E113552 Type 2 diabetes mellitus with stable proliferative diabetic retinopathy, left eye

E113553 Type 2 diabetes mellitus with stable proliferative diabetic retinopathy, bilateral

E113559 Type 2 diabetes mellitus with stable proliferative diabetic retinopathy, unspecified eye

E11359 Type 2 diabetes mellitus with proliferative diabetic retinopathy without macular edema

E113591 Type 2 diabetes mellitus with proliferative diabetic retinopathy without macular edema, right eye

E113592 Type 2 diabetes mellitus with proliferative diabetic retinopathy without macular edema, left eye

E113593 Type 2 diabetes mellitus with proliferative diabetic retinopathy without macular edema, bilateral

(continued on next page) 
E113599 Type 2 diabetes mellitus with proliferative diabetic retinopathy without macular edema, unspecified eye

E1136 Type 2 diabetes mellitus with diabetic cataract

E1137 $\times 1$ Type 2 diabetes mellitus with diabetic macular edema, resolved following treatment, right eye

E1137 $\times 2$ Type 2 diabetes mellitus with diabetic macular edema, resolved following treatment, left eye

E1137 $\times 3$ Type 2 diabetes mellitus with diabetic macular edema, resolved following treatment, bilateral

E1137 $\times 9$ Type 2 diabetes mellitus with diabetic macular edema, resolved following treatment, unspecified eye

E1139 Type 2 diabetes mellitus with other diabetic ophthalmic complication

E1140 Type 2 diabetes mellitus with diabetic neuropathy, unspecified

E1141 Type 2 diabetes mellitus with diabetic mononeuropathy

E1142 Type 2 diabetes mellitus with diabetic polyneuropathy

E1143 Type 2 diabetes mellitus with diabetic autonomic (poly)neuropathy

E1144 Type 2 diabetes mellitus with diabetic amyotrophy

E1149 Type 2 diabetes mellitus with other diabetic neurologic complication

E1151 Type 2 diabetes mellitus with diabetic peripheral angiopathy without gangrene

E1152 Type 2 diabetes mellitus with diabetic peripheral angiopathy with gangrene

E1159 Type 2 diabetes mellitus with other circulatory complications

E11610 Type 2 diabetes mellitus with diabetic neuropathic arthropathy

E11618 Type 2 diabetes mellitus with other diabetic arthropathy

E11620 Type 2 diabetes mellitus with diabetic dermatitis

E11621 Type 2 diabetes mellitus with foot ulcer

E11622 Type 2 diabetes mellitus with other skin ulcer

E11628 Type 2 diabetes mellitus with other skin complications

E11630 Type 2 diabetes mellitus with periodontal disease

E11638 Type 2 diabetes mellitus with other oral complications

E11641 Type 2 diabetes mellitus with hypoglycemia with coma

E11649 Type 2 diabetes mellitus with hypoglycemia without coma

E1165 Type 2 diabetes mellitus with hyperglycemia

E1169 Type 2 diabetes mellitus with other specified complication

E118 Type 2 diabetes mellitus with unspecified complications

E119 Type 2 diabetes mellitus without complications

E1300 Other specified diabetes mellitus with hyperosmolarity without nonketotic hyperglycemic-hyperosmolar coma (NKHHC)

E1301 Other specified diabetes mellitus with hyperosmolarity with coma

E1310 Other specified diabetes mellitus with ketoacidosis without coma

E1311 Other specified diabetes mellitus with ketoacidosis with coma

E1321 Other specified diabetes mellitus with diabetic nephropathy

E1322 Other specified diabetes mellitus with diabetic chronic kidney disease

E1329 Other specified diabetes mellitus with other diabetic kidney complication

E13311 Other specified diabetes mellitus with unspecified diabetic retinopathy with macular edema

E13319 Other specified diabetes mellitus with unspecified diabetic retinopathy without macular edema

E13321 Other specified diabetes mellitus with mild nonproliferative diabetic retinopathy with macular edema

E133211 Other specified diabetes mellitus with mild nonproliferative diabetic retinopathy with macular edema, right eye

E133212 Other specified diabetes mellitus with mild nonproliferative diabetic retinopathy with macular edema, left eye

E133213 Other specified diabetes mellitus with mild nonproliferative diabetic retinopathy with macular edema, bilateral

E133219 Other specified diabetes mellitus with mild nonproliferative diabetic retinopathy with macular edema, unspecified eye

E13329 Other specified diabetes mellitus with mild nonproliferative diabetic retinopathy without macular edema 
E133291 Other specified diabetes mellitus with mild nonproliferative diabetic retinopathy without macular edema, right eye E133292 Other specified diabetes mellitus with mild nonproliferative diabetic retinopathy without macular edema, left eye E133293 Other specified diabetes mellitus with mild nonproliferative diabetic retinopathy without macular edema, bilateral

E133299 Other specified diabetes mellitus with mild nonproliferative diabetic retinopathy without macular edema, unspecified eye

E13331 Other specified diabetes mellitus with moderate nonproliferative diabetic retinopathy with macular edema

E133311 Other specified diabetes mellitus with moderate nonproliferative diabetic retinopathy with macular edema, right eye

E133312 Other specified diabetes mellitus with moderate nonproliferative diabetic retinopathy with macular edema, left eye

E133313 Other specified diabetes mellitus with moderate nonproliferative diabetic retinopathy with macular edema, bilateral

E133319 Other specified diabetes mellitus with moderate nonproliferative diabetic retinopathy with macular edema, unspecified eye

E13339 Other specified diabetes mellitus with moderate nonproliferative diabetic retinopathy without macular edema

E133391 Other specified diabetes mellitus with moderate nonproliferative diabetic retinopathy without macular edema, right eye

E133392 Other specified diabetes mellitus with moderate nonproliferative diabetic retinopathy without macular edema, left eye E133393 Other specified diabetes mellitus with moderate nonproliferative diabetic retinopathy without macular edema, bilateral

E133399 Other specified diabetes mellitus with moderate nonproliferative diabetic retinopathy without macular edema, unspecified eye

E13341 Other specified diabetes mellitus with severe nonproliferative diabetic retinopathy with macular edema

E133411 Other specified diabetes mellitus with severe nonproliferative diabetic retinopathy with macular edema, right eye

E133412 Other specified diabetes mellitus with severe nonproliferative diabetic retinopathy with macular edema, left eye

E133413 Other specified diabetes mellitus with severe nonproliferative diabetic retinopathy with macular edema, bilateral

E133419 Other specified diabetes mellitus with severe nonproliferative diabetic retinopathy with macular edema, unspecified eye

E13349 Other specified diabetes mellitus with severe nonproliferative diabetic retinopathy without macular edema

E133491 Other specified diabetes mellitus with severe nonproliferative diabetic retinopathy without macular edema, right eye

E133492 Other specified diabetes mellitus with severe nonproliferative diabetic retinopathy without macular edema, left eye

E133493 Other specified diabetes mellitus with severe nonproliferative diabetic retinopathy without macular edema, bilateral

E133499 Other specified diabetes mellitus with severe nonproliferative diabetic retinopathy without macular edema, unspecified eye

E13351 Other specified diabetes mellitus with proliferative diabetic retinopathy with macular edema

E133511 Other specified diabetes mellitus with proliferative diabetic retinopathy with macular edema, right eye

E133512 Other specified diabetes mellitus with proliferative diabetic retinopathy with macular edema, left eye

E133513 Other specified diabetes mellitus with proliferative diabetic retinopathy with macular edema, bilateral

E133519 Other specified diabetes mellitus with proliferative diabetic retinopathy with macular edema, unspecified eye

E133521 Other specified diabetes mellitus with proliferative diabetic retinopathy with traction retinal detachment involving the macula, right eye

E133522 Other specified diabetes mellitus with proliferative diabetic retinopathy with traction retinal detachment involving the macula, left eye

E133523 Other specified diabetes mellitus with proliferative diabetic retinopathy with traction retinal detachment involving the macula, bilateral

E133529 Other specified diabetes mellitus with proliferative diabetic retinopathy with traction retinal detachment involving the macula, unspecified eye

(continued on next page) 
E133531 Other specified diabetes mellitus with proliferative diabetic retinopathy with traction retinal detachment not involving the macula, right eye

E133532 Other specified diabetes mellitus with proliferative diabetic retinopathy with traction retinal detachment not involving the macula, left eye

E133533 Other specified diabetes mellitus with proliferative diabetic retinopathy with traction retinal detachment not involving the macula, bilateral

E133539 Other specified diabetes mellitus with proliferative diabetic retinopathy with traction retinal detachment not involving the macula, unspecified eye

E133541 Other specified diabetes mellitus with proliferative diabetic retinopathy with combined traction retinal detachment and rhegmatogenous retinal detachment, right eye

E133542 Other specified diabetes mellitus with proliferative diabetic retinopathy with combined traction retinal detachment and rhegmatogenous retinal detachment, left eye

E133543 Other specified diabetes mellitus with proliferative diabetic retinopathy with combined traction retinal detachment and rhegmatogenous retinal detachment, bilateral

E133549 Other specified diabetes mellitus with proliferative diabetic retinopathy with combined traction retinal detachment and rhegmatogenous retinal detachment, unspecified eye

E133551 Other specified diabetes mellitus with stable proliferative diabetic retinopathy, right eye

E133552 Other specified diabetes mellitus with stable proliferative diabetic retinopathy, left eye

E133553 Other specified diabetes mellitus with stable proliferative diabetic retinopathy, bilateral

E133559 Other specified diabetes mellitus with stable proliferative diabetic retinopathy, unspecified eye

E13359 Other specified diabetes mellitus with proliferative diabetic retinopathy without macular edema

E133591 Other specified diabetes mellitus with proliferative diabetic retinopathy without macular edema, right eye

E133592 Other specified diabetes mellitus with proliferative diabetic retinopathy without macular edema, left eye

E133593 Other specified diabetes mellitus with proliferative diabetic retinopathy without macular edema, bilateral

E133599 Other specified diabetes mellitus with proliferative diabetic retinopathy without macular edema, unspecified eye

E1336 Other specified diabetes mellitus with diabetic cataract

E1337 $\times 1$ Other specified diabetes mellitus with diabetic macular edema, resolved following treatment, right eye

E1337 $\times 2$ Other specified diabetes mellitus with diabetic macular edema, resolved following treatment, left eye

E1337 $\times 3$ Other specified diabetes mellitus with diabetic macular edema, resolved following treatment, bilateral

E1337 $\times 9$ Other specified diabetes mellitus with diabetic macular edema, resolved following treatment, unspecified eye

E1339 Other specified diabetes mellitus with other diabetic ophthalmic complication

E1340 Other specified diabetes mellitus with diabetic neuropathy, unspecified

E1341 Other specified diabetes mellitus with diabetic mononeuropathy

E1342 Other specified diabetes mellitus with diabetic polyneuropathy

E1343 Other specified diabetes mellitus with diabetic autonomic (poly)neuropathy

E1344 Other specified diabetes mellitus with diabetic amyotrophy

E1349 Other specified diabetes mellitus with other diabetic neurologic complication

E1351 Other specified diabetes mellitus with diabetic peripheral angiopathy without gangrene

E1352 Other specified diabetes mellitus with diabetic peripheral angiopathy with gangrene

E1359 Other specified diabetes mellitus with other circulatory complications

E13610 Other specified diabetes mellitus with diabetic neuropathic arthropathy

E13618 Other specified diabetes mellitus with other diabetic arthropathy

E13620 Other specified diabetes mellitus with diabetic dermatitis

E13621 Other specified diabetes mellitus with foot ulcer

E13622 Other specified diabetes mellitus with other skin ulcer

E13628 Other specified diabetes mellitus with other skin complications

E13630 Other specified diabetes mellitus with periodontal disease

E13638 Other specified diabetes mellitus with other oral complications

(continued on next page) 
E13641 Other specified diabetes mellitus with hypoglycemia with coma

E13649 Other specified diabetes mellitus with hypoglycemia without coma

E1365 Other specified diabetes mellitus with hyperglycemia

E1369 Other specified diabetes mellitus with other specified complication

E138 Other specified diabetes mellitus with unspecified complications

E139 Other specified diabetes mellitus without complications

\section{Diabetes Diagnosis Codes: Other}

Note: This category includes all diabetes cases for which there is no amputation and that do not fit elsewhere.

E109 Type 1 diabetes mellitus without complications

E11618 Type 2 diabetes mellitus with other diabetic arthropathy

E119 Type 2 diabetes mellitus without complications

E1300 Other specified diabetes mellitus with hyperosmolarity without nonketotic hyperglycemic-hyperosmolar coma (NKHHC)

E1301 Other specified diabetes mellitus with hyperosmolarity with coma

E1310 Other specified diabetes mellitus with ketoacidosis without coma

E1311 Other specified diabetes mellitus with ketoacidosis with coma

E1321 Other specified diabetes mellitus with diabetic nephropathy

E1322 Other specified diabetes mellitus with diabetic chronic kidney disease

E1329 Other specified diabetes mellitus with other diabetic kidney complication

E13311 Other specified diabetes mellitus with unspecified diabetic retinopathy with macular edema

E13319 Other specified diabetes mellitus with unspecified diabetic retinopathy without macular edema

E13321 Other specified diabetes mellitus with mild nonproliferative diabetic retinopathy with macular edema

E133211 Other specified diabetes mellitus with mild nonproliferative diabetic retinopathy with macular edema, right eye

E133212 Other specified diabetes mellitus with mild nonproliferative diabetic retinopathy with macular edema, left eye

E133213 Other specified diabetes mellitus with mild nonproliferative diabetic retinopathy with macular edema, bilateral

E133219 Other specified diabetes mellitus with mild nonproliferative diabetic retinopathy with macular edema, unspecified eye

E13329 Other specified diabetes mellitus with mild nonproliferative diabetic retinopathy without macular edema

E133291 Other specified diabetes mellitus with mild nonproliferative diabetic retinopathy without macular edema, right eye

E133292 Other specified diabetes mellitus with mild nonproliferative diabetic retinopathy without macular edema, left eye

E133293 Other specified diabetes mellitus with mild nonproliferative diabetic retinopathy without macular edema, bilateral E133299 Other specified diabetes mellitus with mild nonproliferative diabetic retinopathy without macular edema, unspecified eye

E13331 Other specified diabetes mellitus with moderate nonproliferative diabetic retinopathy with macular edema

E133311 Other specified diabetes mellitus with moderate nonproliferative diabetic retinopathy with macular edema, right eye E133312 Other specified diabetes mellitus with moderate nonproliferative diabetic retinopathy with macular edema, left eye E133313 Other specified diabetes mellitus with moderate nonproliferative diabetic retinopathy with macular edema, bilateral

E133319 Other specified diabetes mellitus with moderate nonproliferative diabetic retinopathy with macular edema, unspecified eye

E13339 Other specified diabetes mellitus with moderate nonproliferative diabetic retinopathy without macular edema

E133391 Other specified diabetes mellitus with moderate nonproliferative diabetic retinopathy without macular edema, right eye

E133392 Other specified diabetes mellitus with moderate nonproliferative diabetic retinopathy without macular edema, left eye E133393 Other specified diabetes mellitus with moderate nonproliferative diabetic retinopathy without macular edema, bilateral 
E133399 Other specified diabetes mellitus with moderate nonproliferative diabetic retinopathy without macular edema, unspecified eye

E13341 Other specified diabetes mellitus with severe nonproliferative diabetic retinopathy with macular edema

E133411 Other specified diabetes mellitus with severe nonproliferative diabetic retinopathy with macular edema, right eye

E133412 Other specified diabetes mellitus with severe nonproliferative diabetic retinopathy with macular edema, left eye

E133413 Other specified diabetes mellitus with severe nonproliferative diabetic retinopathy with macular edema, bilateral

E133419 Other specified diabetes mellitus with severe nonproliferative diabetic retinopathy with macular edema, unspecified eye

E13349 Other specified diabetes mellitus with severe nonproliferative diabetic retinopathy without macular edema

E133491 Other specified diabetes mellitus with severe nonproliferative diabetic retinopathy without macular edema, right eye

E133492 Other specified diabetes mellitus with severe nonproliferative diabetic retinopathy without macular edema, left eye

E133493 Other specified diabetes mellitus with severe nonproliferative diabetic retinopathy without macular edema, bilateral E133499 Other specified diabetes mellitus with severe nonproliferative diabetic retinopathy without macular edema, unspecified eye

E13351 Other specified diabetes mellitus with proliferative diabetic retinopathy with macular edema

E133511 Other specified diabetes mellitus with proliferative diabetic retinopathy with macular edema, right eye

E133512 Other specified diabetes mellitus with proliferative diabetic retinopathy with macular edema, left eye

E133513 Other specified diabetes mellitus with proliferative diabetic retinopathy with macular edema, bilateral

E133519 Other specified diabetes mellitus with proliferative diabetic retinopathy with macular edema, unspecified eye

E133521 Other specified diabetes mellitus with proliferative diabetic retinopathy with traction retinal detachment involving the macula, right eye

E133522 Other specified diabetes mellitus with proliferative diabetic retinopathy with traction retinal detachment involving the macula, left eye

E133523 Other specified diabetes mellitus with proliferative diabetic retinopathy with traction retinal detachment involving the macula, bilateral

E133529 Other specified diabetes mellitus with proliferative diabetic retinopathy with traction retinal detachment involving the macula, unspecified eye

E133531 Other specified diabetes mellitus with proliferative diabetic retinopathy with traction retinal detachment not involving the macula, right eye

E133532 Other specified diabetes mellitus with proliferative diabetic retinopathy with traction retinal detachment not involving the macula, left eye

E133533 Other specified diabetes mellitus with proliferative diabetic retinopathy with traction retinal detachment not involving the macula, bilateral

E133539 Other specified diabetes mellitus with proliferative diabetic retinopathy with traction retinal detachment not involving the macula, unspecified eye

E133541 Other specified diabetes mellitus with proliferative diabetic retinopathy with combined traction retinal detachment and rhegmatogenous retinal detachment, right eye

E133542 Other specified diabetes mellitus with proliferative diabetic retinopathy with combined traction retinal detachment and rhegmatogenous retinal detachment, left eye

E133543 Other specified diabetes mellitus with proliferative diabetic retinopathy with combined traction retinal detachment and rhegmatogenous retinal detachment, bilateral

E133549 Other specified diabetes mellitus with proliferative diabetic retinopathy with combined traction retinal detachment and rhegmatogenous retinal detachment, unspecified eye

E133551 Other specified diabetes mellitus with stable proliferative diabetic retinopathy, right eye

E133552 Other specified diabetes mellitus with stable proliferative diabetic retinopathy, left eye

E133553 Other specified diabetes mellitus with stable proliferative diabetic retinopathy, bilateral

E133559 Other specified diabetes mellitus with stable proliferative diabetic retinopathy, unspecified eye

E13359 Other specified diabetes mellitus with proliferative diabetic retinopathy without macular edema

E133591 Other specified diabetes mellitus with proliferative diabetic retinopathy without macular edema, right eye

(continued on next page) 
E133592 Other specified diabetes mellitus with proliferative diabetic retinopathy without macular edema, left eye E133593 Other specified diabetes mellitus with proliferative diabetic retinopathy without macular edema, bilateral E133599 Other specified diabetes mellitus with proliferative diabetic retinopathy without macular edema, unspecified eye

E1336 Other specified diabetes mellitus with diabetic cataract

E1337 $\times 1$ Other specified diabetes mellitus with diabetic macular edema, resolved following treatment, right eye

E1337 $\times 2$ Other specified diabetes mellitus with diabetic macular edema, resolved following treatment, left eye

E1337 $\times 3$ Other specified diabetes mellitus with diabetic macular edema, resolved following treatment, bilateral

E1337 $\times 9$ Other specified diabetes mellitus with diabetic macular edema, resolved following treatment, unspecified eye

E1339 Other specified diabetes mellitus with other diabetic ophthalmic complication

E1340 Other specified diabetes mellitus with diabetic neuropathy, unspecified

E1341 Other specified diabetes mellitus with diabetic mononeuropathy

E1342 Other specified diabetes mellitus with diabetic polyneuropathy

E1343 Other specified diabetes mellitus with diabetic autonomic (poly)neuropathy

E1344 Other specified diabetes mellitus with diabetic amyotrophy

E1349 Other specified diabetes mellitus with other diabetic neurologic complication

E1351 Other specified diabetes mellitus with diabetic peripheral angiopathy without gangrene

E1352 Other specified diabetes mellitus with diabetic peripheral angiopathy with gangrene

E1359 Other specified diabetes mellitus with other circulatory complications

E13610 Other specified diabetes mellitus with diabetic neuropathic arthropathy

E13618 Other specified diabetes mellitus with other diabetic arthropathy

E13620 Other specified diabetes mellitus with diabetic dermatitis

E13621 Other specified diabetes mellitus with foot ulcer

E13622 Other specified diabetes mellitus with other skin ulcer

E13628 Other specified diabetes mellitus with other skin complications

E13630 Other specified diabetes mellitus with periodontal disease

E13638 Other specified diabetes mellitus with other oral complications

E13641 Other specified diabetes mellitus with hypoglycemia with coma

E13649 Other specified diabetes mellitus with hypoglycemia without coma

E1365 Other specified diabetes mellitus with hyperglycemia

E1369 Other specified diabetes mellitus with other specified complication

E138 Other specified diabetes mellitus with unspecified complications

E139 Other specified diabetes mellitus without complications

\title{
Diabetes Diagnosis Codes: Unclassified
}

Note: This category includes all diabetes codes that do not fall in any of the other existing diabetes categories.

E100, E1012, E1020, E1023, E1028, E1030, E1050, E1060, E1068, E1070, E1071, E1078, E110, E1110, E1111, E1112, E1120, E1123,

E1128, E1130, E1138, E1150, E1160, E1168, E1170, E1171, E1178, E130, E1312, E1323, E1328, E1368, E1370

Data from Agency for Healthcare Research and Quality. Prevention Quality Indicator Technical Specifications. Available at: http://www.qualityindicators.ahrq.gov/Modules/PQI_TechSpec_ICD10_v70.aspx. Accessed October 20, 2019.

\section{Common Nonpreventable Clinical Diagnosis Groups and Respective ICD-10 Codes Used to Indicate Diagnosis}

\author{
Sepsis
}

A40 Streptococcal septicaemia

A41 Other septicaemia

R65 Systemic Inflammatory Response Syndrome [SIRS]

(continued on next page) 


\section{Stroke, Transient Ischemic Attack, Intracranial Hemorrhage}

I60 Subarachnoid hemorrhage

I61 Intracerebral hemorrhage

I62 Other nontraumatic intracranial hemorrhage

I63 Cerebral infarction

I64 Stroke, not specified as hemorrhage or infarction

I65 Occlusion and stenosis of precerebral arteries, not resulting in cerebral infarction

I66 Occlusion and stenosis of cerebral arteries, not resulting in cerebral infarction

G45 Transient cerebral ischemic attacks and related syndromes

S06.6 Traumatic subarachnoid hemorrhage

\section{Cancer and Chemotherapy}

C00-C97 Malignant neoplasms

No code for chemotherapy

\section{Cardiac Valve Disease}

I05-I08 Chronic rheumatic heart diseases

\section{Arrhythmia}

I44 Atrioventricular and left bundle-branch block

I45 Other conduction disorders

I46 Cardiac arrest

I47 Paroxysmal tachycardia

I48 Atrial fibrillation and flutter

I49 Other cardiac arrhythmias

Ischemic Heart Disease

I20-I25 Ischemic heart diseases

\section{Dementia}

F00-F03 Dementia

G30 Alzheimer's disease

\section{Gastrointestinal Infections and Disorders}

A08 Viral and other specified intestinal infections

K20-K31 Diseases of esophagus, stomach, and duodenum

K55-K63 Other diseases of intestines

K65-K67 Diseases of peritoneum

Kidney Failure

N00-N08 Glomerular diseases

N10-N16 Renal tubulo-interstitial diseases

N17-N19 Renal failure

\section{Cellulitis}

L02 Cutaneous abscess, furuncle, and carbuncle

L03 Cellulitis 


\section{Orthopedic Conditions}

M00 Pyogenic arthritis

M01 Direct infections of joint in infectious and parasitic diseases classified elsewhere

M02 Reactive arthropathies

M05 Seropositive rheumatoid arthritis

M06 Other rheumatoid arthritis

M08 Juvenile arthritis

M11 Other crystal arthropathies

M12 Other specific arthropathies

M13 Other arthritis

M14 Arthropathies in other diseases classified elsewhere

M15 Polyarthrosis

M19 Other arthrosis

M20 Acquired deformities of fingers and toes

M21 Other acquired deformities of limbs

M22 Disorders of patella

M23 Internal derangement of knee

M24 Other specific joint derangements

M25 Other joint disorders, not elsewhere classified

M32 Systemic lupus erythematosus

M33 Dermatopolymyositis

M35 Other systemic involvement of connective tissue

M42 Spinal osteochondrosis

M43 Other deforming dorsopathies

M45 Ankylosing spondylitis

M46 Other inflammatory spondylopathies

M47 Spondylosis

M48 Other spondylopathies

M49 Spondylopathies in diseases classified elsewhere

M50 Cervical disc disorders

M51 Other intervertebral disc disorders

M53 Other dorsopathies, not elsewhere classified

M54 Dorsalgia

M60 Myositis

M62 Other disorders of muscle

M63 Disorders of muscle in diseases classified elsewhere

M65 Synovitis and tenosynovitis

M66 Spontaneous rupture of synovium and tendon

M67 Other disorders of synovium and tendon

M68 Disorders of synovium and tendon in diseases classified elsewhere

M70 Soft tissue disorders related to use, overuse, and pressure

M71 Other bursopathies

M72 Fibroblastic disorders

M73 Soft tissue disorders in diseases classified elsewhere

M75 Shoulder lesions

M76 Enthesopathies of lower limb, excluding foot 
M77 Other enthesopathies

M79 Other soft tissue disorders, not elsewhere classified

M80 Osteoporosis with pathologic fracture

M81 Osteoporosis without pathologic fracture

M82 Osteoporosis in diseases classified elsewhere

M84 Pathologic fracture, not elsewhere classified

M86 Osteomyelitis

M88 Paget's disease of bone (osteitis deformans)

M89 Other disorders of bone

M90 Osteopathies in diseases classified elsewhere

M91 Juvenile osteochondrosis of hip and pelvis

M92 Other juvenile osteochondrosis

M93 Other osteochondropathies

M99 Biomechanical lesions, not elsewhere classified

R29 Other symptoms and signs involving the nervous and musculoskeletal systems

S12 Fracture of neck

S14 Injury of nerves and spinal cord at neck level

S22 Fracture of rib(s), sternum, and thoracic spine

S24 Injury of nerves and spinal cord at thorax level

S32 Fracture of lumbar spine and pelvis

S34 Injury of nerves and lumbar spinal cord at abdomen, lower back, and pelvis level

S72 Fracture of femur

Syncope and Dizziness

R55 Syncope and collapse

R42 Dizziness and giddiness

Other Pulmonary

J44 Other chronic obstructive pulmonary disease

J69 Pneumonitis due to solids and liquids

J91 Pleural effusion in conditions classified elsewhere

J96 Respiratory failure, not elsewhere classified

Peripheral Atherosclerosis

I70 Atherosclerosis

K55 Vascular disorders of intestine

\section{Pulmonary Embolism and Deep Vein Thrombosis}

I26 Pulmonary embolism

I27 Other pulmonary heart diseases

182.4 Acute embolism and thrombosis of deep veins of lower extremity

\section{Procedure or Device Complications}

T82 Complications of cardiac and vascular prosthetic devices, implants, and grafts

T83 Complications of genitourinary prosthetic devices, implants, and grafts

T84 Complications of internal orthopedic prosthetic devices, implants, and grafts

T85 Complications of other internal prosthetic devices, implants, and grafts 


\section{Rehabilitation}

Z50 Care involving use of rehabilitation procedures

Data from Joynt KE, Gawande AA, Orav EJ, et al. Contribution of preventable acute care spending to total spending for high-cost Medicare patients. JAMA 2013;309:2572-2578. 